\title{
1 Measurement of the Earth tides with a MEMS gravimeter
}

\author{
2 R. P. Middlemiss, ${ }^{*} \dagger$ A. Samarelli, ${ }^{*}$ D. J. Paul, ${ }^{\dagger}$ J. Hough, ${ }^{*}$ S. Rowan* and G. D. Hammond*
}

3

4

5

6

7

8

9

The ability to measure tiny variations in the local gravitational acceleration allows - amongst other applications - the detection of hidden hydrocarbon reserves, magma build-up before volcanic eruptions, and subterranean tunnels. Several technologies are available that achieve the sensitivities required for such applications (tens of $\mu \mathrm{Gal} / \sqrt{H z}$ ): free-fall gravimeters ${ }^{1}$, spring-based gravimeters $^{2,3}$, superconducting gravimeters ${ }^{4}$, and atom interferometers ${ }^{5}$. All of these devices can observe the Earth Tides ${ }^{6}$; the elastic deformation of the Earth's crust as a result of tidal forces. This is a universally predictable gravitational signal that requires both high sensitivity and high stability over timescales of several days to measure. All present gravimeters, however, have limitations of excessive cost $(>\$ 100 \mathrm{k})$ and high mass $(>8 \mathrm{~kg})$. We have built a microelectromechanical system (MEMS) gravimeter with a sensitivity of $40 \mu \mathrm{Gal} / \sqrt{H z}$ in a package size of only a few cubic centimetres. We demonstrate the remarkable stability and sensitivity of our device with a measurement of the Earth tides. Such a measurement has never been undertaken with a MEMS device, and proves the long term stability of our instrument compared to any other MEMS device, making it the first MEMS accelerometer to transition from seismometer to gravimeter. This heralds a transformative step in MEMS accelerometer technology. MEMS accelerometers - found in most smart phones ${ }^{7}$ - can be mass-produced remarkably cheaply, but most are not sensitive enough,

*S.U.P.A., University of Glasgow, School of Physics and Astronomy, Kelvin Building, University Avenue, Glasgow, G12 8QQ, U.K.

${ }^{\dagger}$ University of Glasgow, School of Engineering, Rankine Building, Oakfield Avenue, Glasgow, G12 8LT, U.K. 
and none have been stable enough to be called a 'gravimeter'. Due to their small size and low cost, MEMS gravimeters could create a new paradigm in gravity mapping: exploration surveys could be carried out with drones instead of low-flying aircraft; they could be used for distributed land surveys in exploration settings, for the monitoring of volcanoes; or built into multi-pixel density contrast imaging arrays.

Gravimeters can be split into two broad categories: absolute gravimeters and relative gravimeters. Absolute gravimeters measure the gravitational acceleration, $g$, by timing a mass in free fall over a set distance. Absolute gravimeters are very accurate but are bulky and expensive. The Micro-g Lacoste FG5 ${ }^{1}$, for example, achieves acceleration sensitivities of $1.6 \mu \mathrm{Gal} / \sqrt{H z}$ (1.6 $\mu \mathrm{Gal} / \sqrt{\mathrm{Hz}}$ is an acceleration measurement of $1.6 \mu \mathrm{Gal}$ in an integration time of 1 second, where $1 \mathrm{Gal}$ is $1 \mathrm{~cm} / \mathrm{s}^{2}$ ), but it costs over $\$ 100 \mathrm{k}$ and weighs $150 \mathrm{~kg}$. Relative gravimeters make gravity measurements relative to the extension of a spring: the deflection of a mass on a spring will change as $g$ varies. These devices can be made smaller than absolute gravimeters but are intrinsically less stable: the spring constant can change with varying environmental conditions. The Scintrex CG5 relative gravimeter (also costing over $\$ 100 \mathrm{k}$, but weighing $8 \mathrm{~kg}$ ) can measure gravity variations down to $2 \mu \mathrm{Ga}^{[2,3]}$ but is much more susceptible to drift than absolute devices. For any mass-on-spring system, increased acceleration sensitivity is achieved by either improving the sensitivity to displacement, or by minimising the ratio, $k / m$, between the spring constant, $k$, and the mass, $m$. A system in which a mass is suspended from a spring within a rigid housing will respond differently to signals above or below the resonance frequency. In the regime below the resonance there will be a linear relationship between the displacement of the proof mass and the acceleration of the housing. This is the region in which the device can be used as an accelerometer/gravimeter. MEMS devices are microscopic mechanical devices made from semiconductor materials. They have the advantage of being mass-producible, light-weight and cheap. Although mobile phone 
accelerometers are not very sensitive, some MEMS devices have been developed that reach sensitivities much better than the $0.23 \mathrm{mGal} / \sqrt{\mathrm{Hz}}$ of the iPhone MEMS device ${ }^{7}$. For example: a device developed by Krishnamoothy et. al. ${ }^{8}$ has a sensitivity of $17 \mu \mathrm{Gal} / \sqrt{H z}$; the SERCEL QuietSieis $^{9}$ has a sensitivity of $15 \mu \mathrm{Gal} / \sqrt{\mathrm{Hz}}$; and a microseismometer developed by Pike et. al.10 has a sensitivity of $2 \mu \mathrm{Gal} / \sqrt{\mathrm{Hz}}$. These devices, however, can only operate as seismometers and do not have a stability sufficient to be classed as gravimeters, which are capable of monitoring low frequency gravimetric signals such as the Earth tides (around $10 \mu \mathrm{Hz}$ ). Table 1 summarises the characteristics of these MEMS seismometers, the Scintrex CG5 gravimeter, and our own gravimeter. Figure 11 provides a further comparison between our own device, the Pike microseismometer ${ }^{10}$, the Scintrex CG5 and two other commercial devices.

The Earth tides are an elastic deformation of the Earth's crust caused by the changing relative phase of the Sun, the Earth and the Moon ${ }^{6}$. They produce a small variation in the local gravitational acceleration, the size of which depends also on the latitude and elevation of the measurement location. Depending on the time of the lunar month, the Earth tides vary in amplitude and frequency, moving between diurnal $\left(2 \times 10^{-5} \mathrm{~Hz}\right)$ and semi-diurnal $\left(1 \times 10^{-5} \mathrm{~Hz}\right)$ peaks. Since the Earth tides have a peak signal strength ${ }^{3}$ of less than $400 \mu \mathrm{Gal}$, and a low frequency oscillation, they are a useful natural signal to demonstrate both the sensitivity and long-term stability of any gravimeter. The Earth tides have never previously been measured with any MEMS device, so a device able to do so will be a transformative step change in the field.

Our device has been designed to have a resonant frequency of under $4 \mathrm{~Hz}$. To achieve such low frequencies a geometric anti-spring system ${ }^{11,12}$ was chosen. With increasing displacement, anti-springs get softer and their resonant frequency gets lower. A geometrical anti-spring requires a pair of arched flexures that meet at a constrained central point. In the case of our MEMS device they meet at the proof mass. This geometry constrains the motion of the proof mass to the axis 


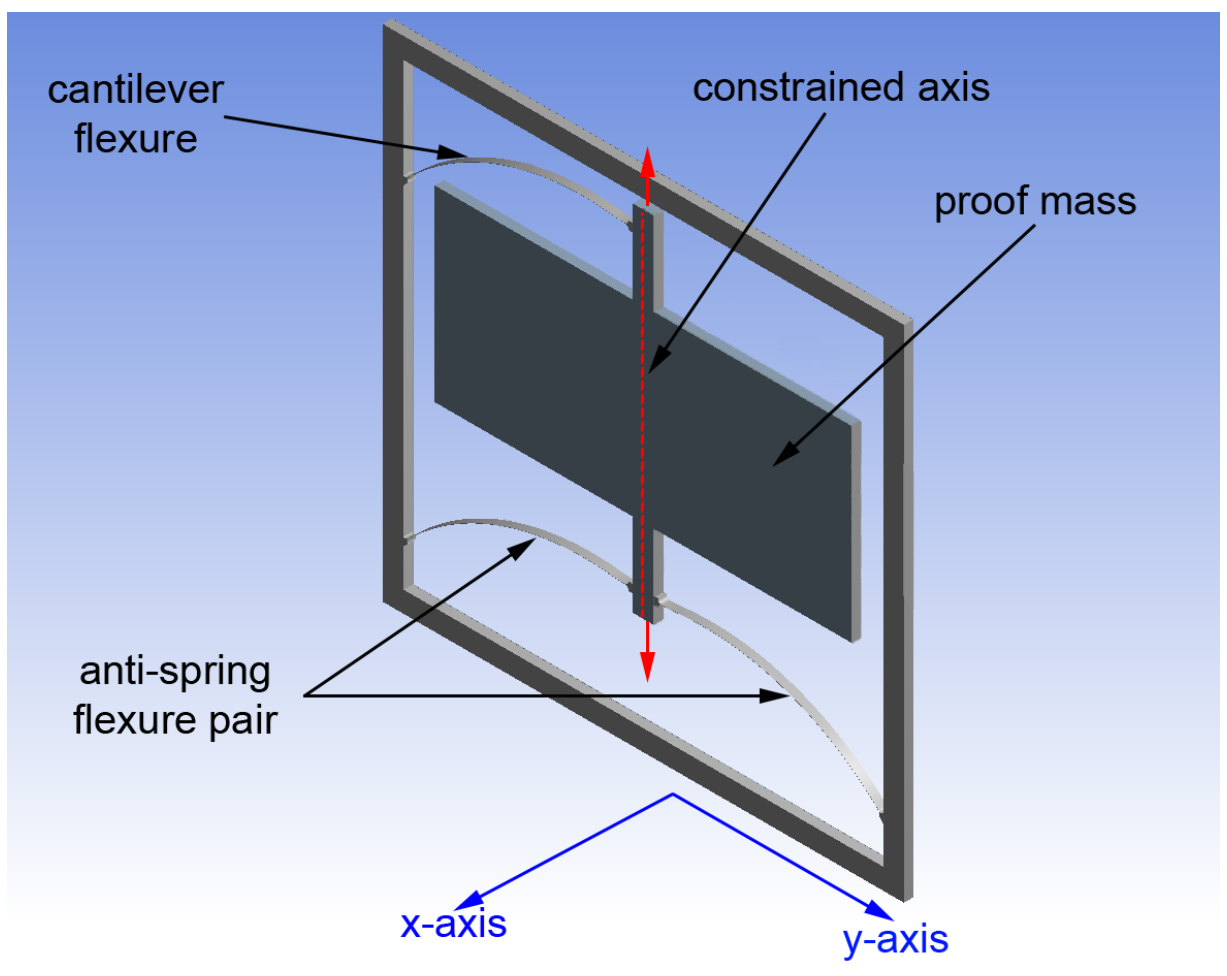

Figure 1

67 shown in Fig. 1. As the proof mass is pulled away from its un-loaded position the spring constant is

lowered. This is in contrast to a Hooke's-law spring, in which the spring constant does not change with increasing displacement. Tilting the MEMS device from horizontal to vertical orientation, pulls the proof mass down, thus lowering the frequency from over $20 \mathrm{~Hz}$ when horizontal to $2.3 \mathrm{~Hz}$ 
$2.3 \mathrm{~Hz}$ is the lowest resonant frequency of any reported MEMS device to date. To our knowledge the next lowest resonant frequency reported is $10.2 \mathrm{~Hz}$ in a device made by Pike et al.13. The fact that the system has a Hooke's law behaviour in its vertical configuration means that it is less sensitive to tilt in the x-axis (see Fig. 1) than would be the case for a normal geometrical anti-spring (see Fig. 12).

The proof mass motion is measured using an optical shadow sensor ${ }^{14}$. Here a light emitting diode (LED) illuminates a photodiode with the MEMS device mounted in between. Motion of the proof mass modulates the shadow, generating a change in the current output of the photodiode. This shadow sensor (Fig. 2) achieves a high sensitivity (equating to an acceleration noise floor of $\leq 10 \mu \mathrm{Gal}$ at the sampling frequency of $0.03 \mathrm{~Hz}$ ), whilst allowing a large dynamic range of up to $50 \mu \mathrm{m}$.

Observation of the Earth tides requires stable operation over several days. The main contributor to parasitic motion is the varying temperatures of the system. For this reason the ' $\mathrm{C}$ '-shaped structure of the shadow sensor was fabricated from fused silica because of its low thermal expansion coefficient at room temperature $\left(4.1 \times 10^{-7} K^{-1}\right)^{15}$. Silicon has a significantly larger thermal expansion coefficient $\left(2.6 \times 10^{-6} K^{-1}\right)^{16}$, but silicon was used to make the MEMS because it is a standard fabrication material in the semiconductor industry, it has high mechanical strength, and its thermal properties are well characterised. The dominant mechanism by which temperature variations affect the gravity measurement is the change in Young's modulus, $Y$, of the flexures 17,18 . This in turn alters the spring constant of the flexures, resulting in a variation of $\mathrm{k}, 1 / k d k / d T$, of $7.88 \times 10^{-6} K^{-1}$. We therefore implemented servo control loops to maintain the temperature of the system to within $1 \mathrm{mK}$. A $1 \mathrm{mK}$ change in temperature would give an uncertainty in the gravity reading of $\sim 25 \mu \mathrm{Gal}$. The primary control loop maintained the temperature of the MEMS device directly, the second controlling the temperature of a copper thermal shield that encased the 


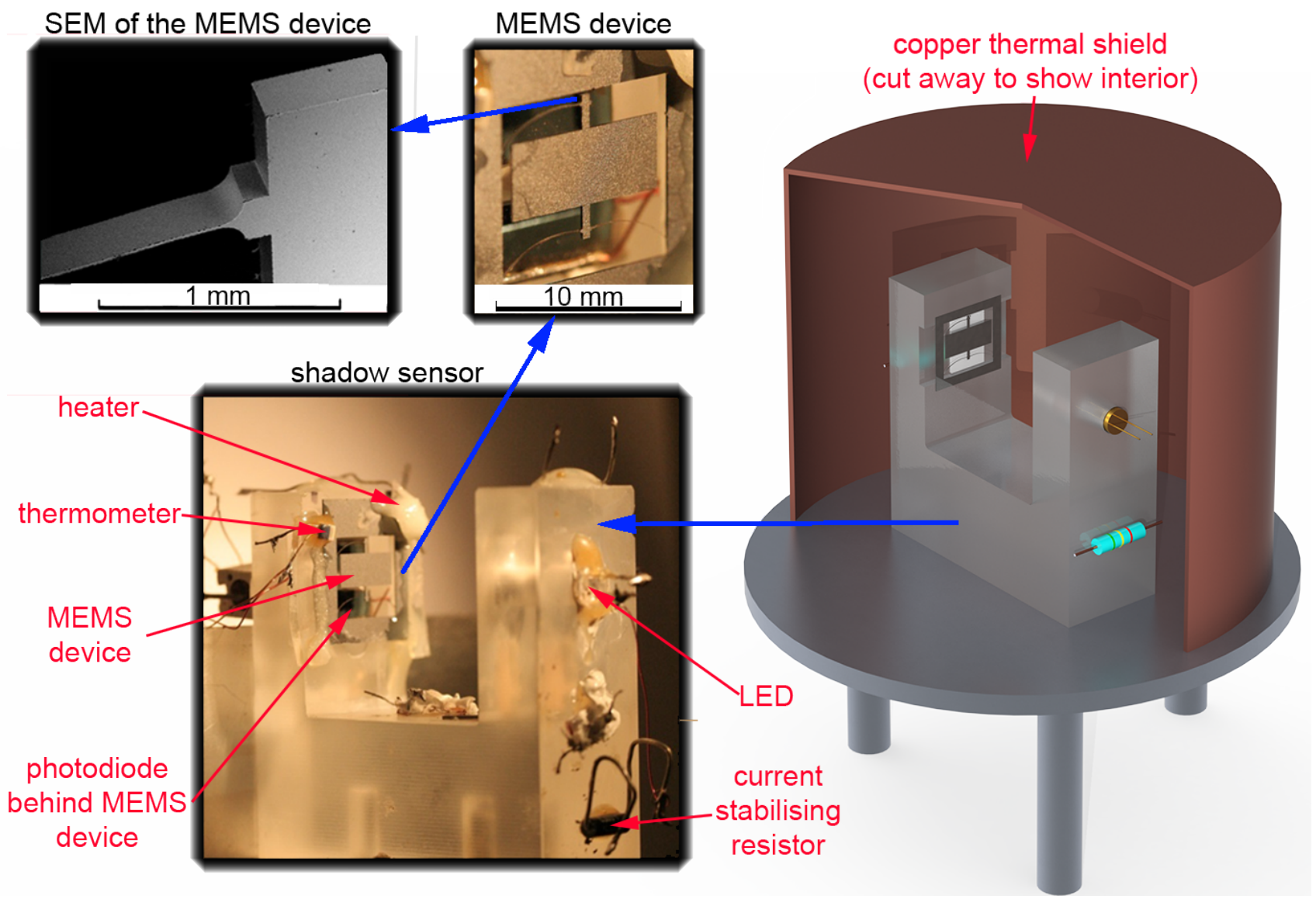

Figure 2 


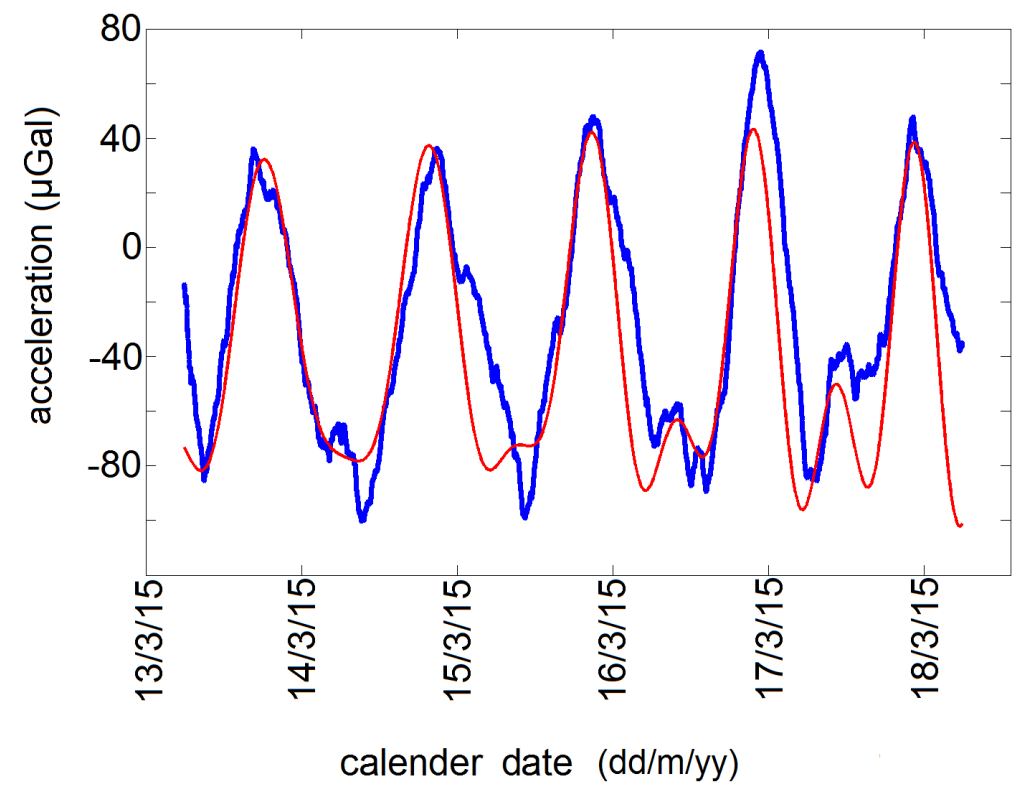

Figure 3

103

entire shadow sensor (Fig. 2). The MEMS device was placed inside a vacuum system. This was bolted to the floor without an external seismic isolation isolation table, which would be a large and expensive addition.

From December 2014 the system was left in continuous operation whilst the servo control was optimised. Figure 3 demonstrates a data run of five days between the 13/03/15 to the 18/03/15 in which gravitational acceleration is plotted against time. The blue data demonstrates our experimental data averaged with a time constant of 240 minutes (the full noise data can be observed in Fig. 6a), together with a data set filtered with a 10 minute time constant (Fig. 6b). The solid red line is a theoretical plot of the Earth tides as should be observed at our location $\left(55.8719^{\circ} \mathrm{N}\right.$, $4.2875^{\circ} \mathrm{W}$ ), and was plotted using $\operatorname{TSOFT}^{19}$. An ocean loading correction is also included in this theoretical plot to account for the effect of nearby tidal waters pressing on the Earth's crust, although the effect is at the level of $5 \%$ for our laboratory. There is a strong correlation coefficient, $R$, of 0.86 between our experimental data and the theory plot. The correlation indicates that this is the first measurement of Earth tides demonstrated by a MEMS device, a landmark result 
for MEMS gravimetry. This measurement provides a natural calibration for the gravimeter, the results of which allow us to determine that the present sensitivity of the device is $40 \mu \mathrm{Gal} / \sqrt{\mathrm{Hz}}$.

We further performed a stability test of the calibration factor for our device by monitoring the tides at two intervals approximately 3 months apart. The calibration remained constant to better than $5 \%$ (Fig. 13).

The noise floor of our device is limited by seismic noise. A theoretical thermal noise floor of under $0.5 \mu \mathrm{Gal} / \sqrt{H z}$ can be calculated, assuming that losses are due to structural damping 2 . This calculation is based upon a measurement of the quality factor, $Q$, of the device under vacuum of $\sim 80$ (the relaxation time of the MEMS device is $\sim 11 \mathrm{~s}$ ). We observe that the $\mathrm{Q}$ reduces as the resonant frequency is lowered (Fig. 7). This behaviour is due to the fact that in geometrical antisprings: as the resonant frequency is lowered, the restoring force becomes comparable to internal friction $^{21}$.

To put the sensitivity of our device into context, $40 \mu \mathrm{Gal} / \sqrt{\mathrm{Hz}}$ is sufficient in 1 second to detect a tunnel with a cross-sectional area of $2 \mathrm{~m}^{2}$ and length of $4 \mathrm{~m}$ at a depth of $2 \mathrm{~m}$; it could be used to find oil reservoirs of $\geq 50 m \times 50 m \times 50 m$ (with a density contrast of 50\%) at a depth of $150 \mathrm{~m}$; a change of $45 \mu \mathrm{Gal}$ was a 'clear precursor' to a volcanic eruption in the Canary Islands in $2011^{222}$. It is accepted that intrusion of new magma into a reservoir precedes volcanic eruptions ${ }^{23}$; continuous micro gravity measurements around volcanoes are a useful tool in monitoring such events ${ }^{24}$. The ratio of ground deformation to change in gravity can be used to monitor magma chambers at depths of several $\mathrm{km}^{25}$.

In figure 3 a linear drift term has been removed from the data. This drift equates to less than $150 \mu \mathrm{Gal}$ per day, a factor of three better than the drift of the Scintrex CG5 (500 $\mu \mathrm{Gal}$ per day). Both we and Scintrex CG5 auto-correct this drift with software. Figure 4 consists of eight subplots demonstrating the drift characteristics on the MEMS device. Figure 4a shows the full-noise tide 


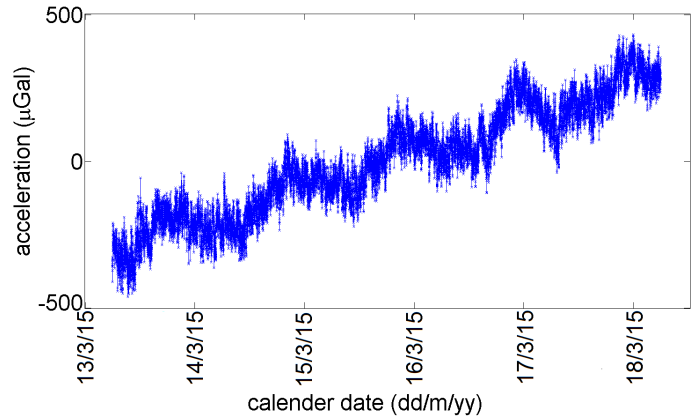

(a)

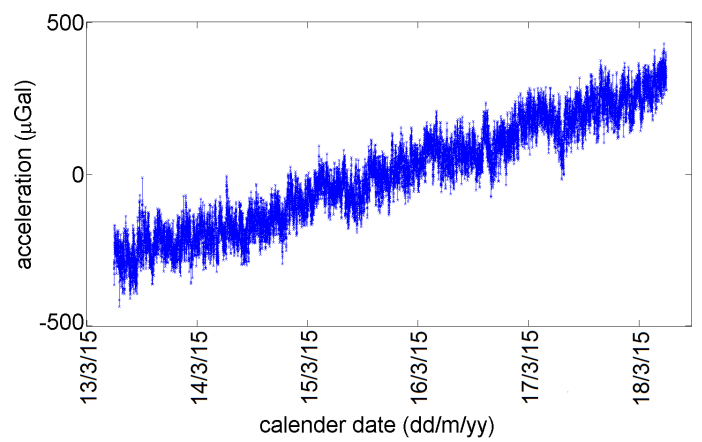

(c)

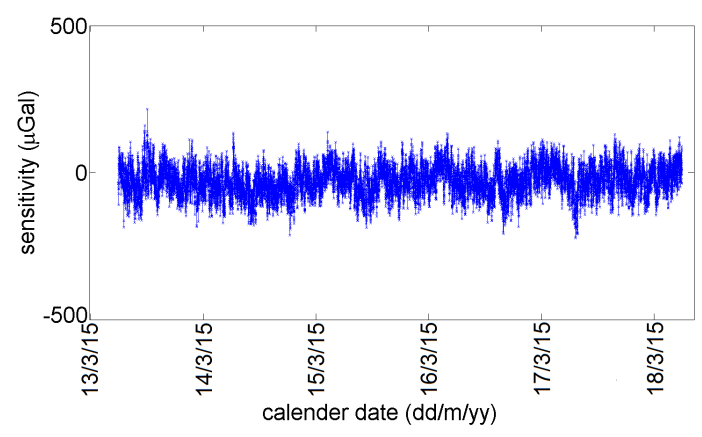

(e)

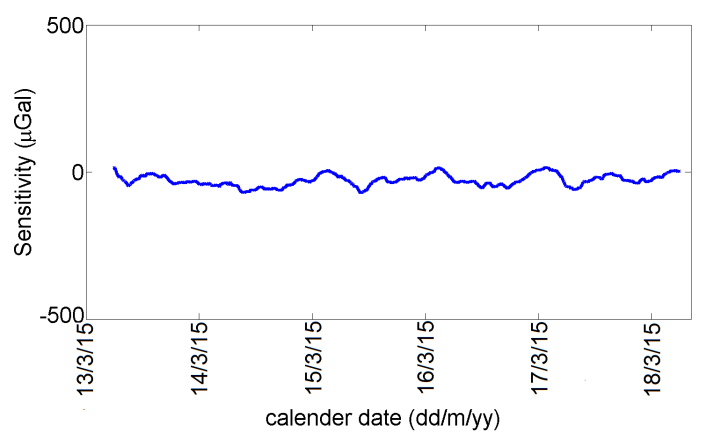

(g)

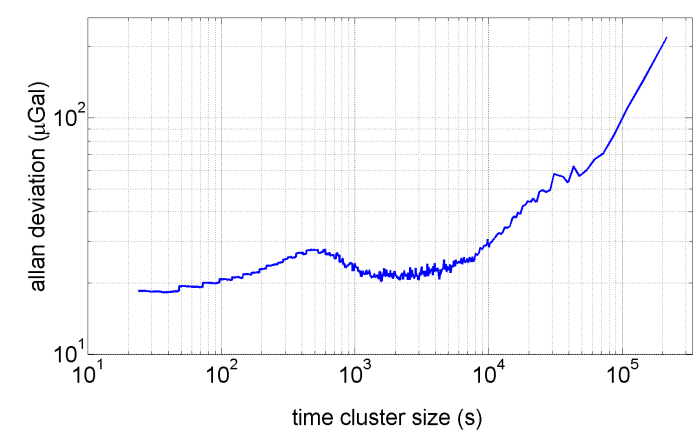

(b)

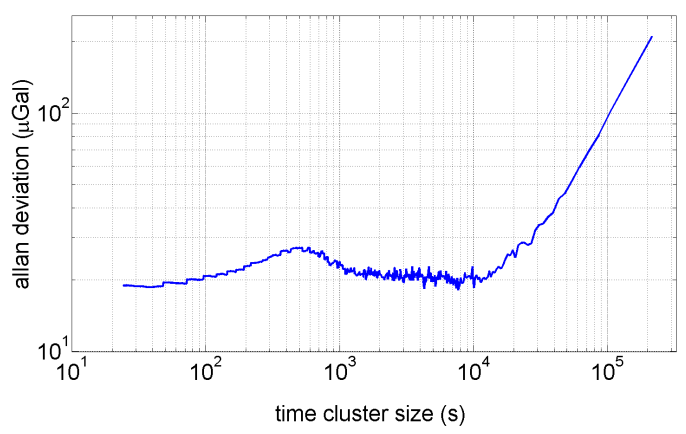

(d)

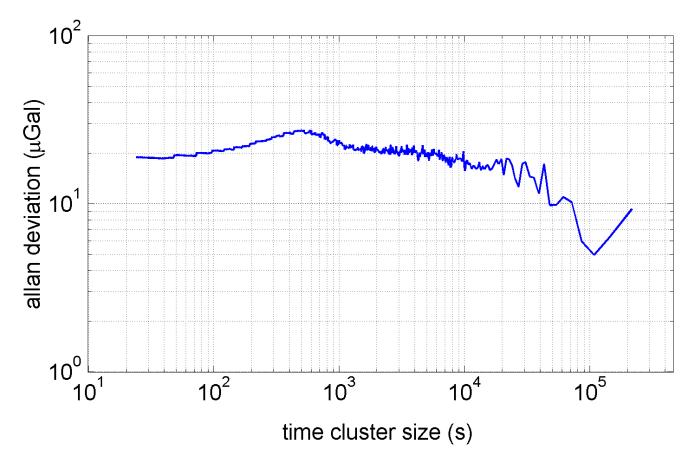

(f)

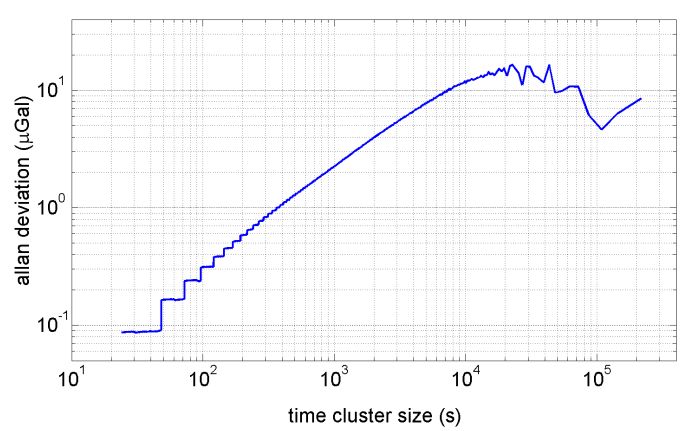

(h)

Figure 4 
data without a linear drift correction. Figure $4 \mathrm{c}$ shows the same data but with the tide signal removed. Figure $4 \mathrm{e}$ shows the same data again but with a linear drift correction. Figures 4b, 4d, $4 \mathrm{f}$ and $4 \mathrm{~h}$ show the Allan deviation for the data in figures 4a, 4c, 4e and 4g respectively. Allan deviation is a technique used to measure the variation over the full frequency range of a signal by averaging over increasingly larger time intervals ${ }^{26}$.

The data analysed in figure 4 spans a frequency range from $10^{-5} \mathrm{~Hz}$ to $0.03 \mathrm{~Hz}$ (the sampling frequency of this data set, which was used to remove the effect of seismic noise). A second data set was taken at a faster sampling rate to observe the response of the device from $0.03 \mathrm{~Hz}$ up to the resonant frequency of $2.3 \mathrm{~Hz}$. Both data sets can be observed in figure 10 in the form of a RMS acceleration sensitivity plot. The Allan deviation for the high frequency series is polluted by the presence of two large signals: the resonant frequency of the device, and the microseismic peak 27,28 . This deviation plot is not a useful measure of the noise of the device and has therefore not been included in figure 4. Figures $4 \mathrm{~b}$ and $4 \mathrm{~d}$ demonstrate the linear drift that the device experiences. Figures 4b, 4d and 4f also demonstrate a small peak at $500 \mathrm{~s}$ that is an artefact of the temperature servo. The broad peak that is only visible on the rising edge of Fig. $4 \mathrm{~b}$ is the tide signal. A comparison between the drift characteristics of our device and some other commercial gravimeters is displayed in figure 11, in which an acceleration power spectral density plot is displayed.

This MEMS device, capable of measuring the Earth tides, represents a significant step forward in the field - it is not just an accelerometer, but a gravimeter. Made from a single silicon chip the size of a postage stamp, this sensor has the lowest reported resonant frequency of any MEMS accelerometer $(2.3 \mathrm{~Hz})$, is within an order of magnitude of the best acceleration sensitivity of any MEMS device $(40 \mu \mathrm{Gal} / \sqrt{\mathrm{Hz}})$, and has the best reported stability of any MEMS device. This prototype will enable the development of a new density contrast imaging technology applicable in many industrial, defence, civil, and environmental applications. It has the potential to be 
inexpensive, mass-produced and lightweight which opens up new markets: it could be flown in drones by oil and gas exploration companies, limiting the need for dangerous low altitude aeroplane flights; it could be used to locate subterranean tunnels; it could be used by building contractors to find underground utilities. Networks of sensors could be operated in unsafe areas for monitoring natural and man-made hazards; for example, on volcanoes or unstable slopes to improve the spatial and temporal resolution of subsurface density changes. This will allow improved hazard forecasting and the reduction of occupational risk to monitoring personnel 25,29 . 


\section{${ }_{172}$ References}

[1] Van Camp, M. Uncertainty of absolute gravity measurements. J. Geophys. Res: Solid Earth 110, B05406 (2005).

[2] Jiang, Z. et al. Relative gravity measurement campaign during the 8th international comparison of absolute gravimeters (2009). Metrologia 49, 95-107 (2012).

[3] Lederer, M. Accuracy of the relative gravity measurement. Acta Geodyn. Geomater. 6, 383$390(2009)$.

[4] Goodkind, J. M. The superconducting gravimeter. Revi. Sci. Instrum. 70, 4131-4152 (1999).

[5] de Angelis, M. et al. Precision gravimetry with atomic sensors. Meas. Sci. and Technol. 20, 022001 (2008).

[6] Farrell, W. E. Earth tides, ocean tides and tidal loading. Philos. T. Roy. Soci. A 274, 253-259 (1973).

[7] D'Alessandro, A. \& D'Anna, G. Suitability of low-cost three-axis MEMS accelerometers in strong-motion seismology: tests on the LIS331DLH (iPhone) accelerometer. B. Seismol. Soc. Am. 103, 2906-2913 (2013).

[8] Krishnamoorthy, U. et al. In-plane MEMS-based nano-g accelerometer with sub-wavelength optical resonant sensor. Sensor. Actuat. A-Phys 145-146, 283-290 (2008).

[9] Lainé, J. \& Mougenot, D. A high-sensitivity MEMS-based accelerometer. The Leading Edge 33, 1234-1242 (2014).

[10] Pike, W. T., Delahunty, A. K., Mukherjee, A. \& Standley, I. M. A self-levelling nano-g silicon seismometer. In Proc. IEEE Sensors 2014, 1599 - 1602 (IEEE, Valencia, 2014). 
[11] Bertolini, A., Cella, G., Desalvo, R. \& Sannibale, V. Seismic noise filters , vertical resonance frequency reduction with geometric anti-springs : a feasibility study. Nucl. Instrum. Meth. A 435, 475-483 (1999).

[12] Cella, G. et al. Seismic attenuation performance of the first prototype of a geometric antispring filter. Nucl. Instrum. Meth. A 487, 652-660 (2002).

[13] Pike, W. T., Standley, I. M. \& Calcutt, S. A Silicon Microseismometer For Mars. In Transducers and Eurosensors XXVII, 622-625 (2013).

[14] Carbone, L. et al. Sensors and actuators for the Advanced LIGO mirror suspensions. Classical Quant. Grav. 29, 115005 (2012).

[15] Bell, C. J., Reid, S. \& Faller, J. Experimental results for nulling the effective thermal expansion coefficient of fused silica fibres under a static stress. Classical Quant. Grav. 31, 065010 (2014).

[16] Hull, R. Properties of Crystalline Silicon (Institution of Engineering and Technology, London, 1999), 1st edn.

[17] Poggi, M. A., McFarland, A. W., Colton, J. S. \& Bottomley, L. A. A method for calculating the spring constant of atomic force microscopy cantilevers with a nonrectangular cross section. Anal. Chem. 77, 1192-1195 (2005).

[18] Cho, C. H. Characterization of Young's modulus of silicon versus temperature using a "beam deflection" method with a four-point bending fixture. Curr. App. Phys. 9, 538-545 (2009).

[19] Van Camp, M. \& Vauterin, P. Tsoft: graphical and interactive software for the analysis of time series and Earth tides. Comput. Geosci. 31, 631-640 (2005). 
[20] Callen, H. B. \& Welton, T. A. Irreversibility and Generalized Noise. Phys. Rev. 83, 34-40 (1951).

[21] Chin, E., Lee, K., Winterflood, J., Ju, L. \& Blair, D. Low frequency vertical geometric anti-spring vibration isolators. Phys. Lett. A 336, 97-105 (2005).

[22] Aparicio, S. S.-M., Sampedro, J. A., Montesinos, F. G. \& Molist, J. M. Volcanic signatures in time gravity variations during the volcanic unrest on El Hierro (Canary Islands). J. Geophys. Res: Solid Earth 119, 5033-5051 (2014).

[23] Mogi, K. Relations between the eruptions of various volcanoes and the deformations of the ground surfaces around them. B. Earthq. Res. I. 36, 99-134 (1958).

[24] Battaglia, M., Gottsmann, J., Carbone, D. \& Fernandez, J. 4D volcano gravimetry. Geophysics 73, WA3-WA18 (2008).

[25] Rymer, H., Williams-Jones, G. \& Keynes, M. Gravity and deformation measurements. Geophys. Res. Lett. 27, 2389-2392 (2000).

[26] Allan, D. Statistics of atomic frequency standards. Proc. IEEE 54, 221-230 (1966).

[27] Peterson, J. Observations and modeling of seismic background noise. U.S. Geological Survey. Open-File Report 93-322 (1993).

[28] Essen, H. H., Kruger, F., Dahm, T. \& Grevemeyer, I. On the generation of secondary microseisms observed in northern and central Europe. J. Geophys. Res: Solid Earth 108, 2506-2520 (2003).

[29] Baxter, P. J. \& Gresham, A. Deaths and injuries in the eruption of Galeras volcano, Colombia, 14 January 1993. J. Volcanol. Geoth. Res. 77, 325-338 (1997). 
234

235

236

[30] Riccardi, U., Rosat, S. \& Hinderer, J. Comparison of the Micro-g LaCoste gPhone-054 spring gravimeter and the GWR-C026 superconducting gravimeter in Strasbourg (France) using a 300-day time series. Metrologia 48, 28-39 (2011). 


\section{Print figure legends}

- Figure 1: The MEMS Device. A figure demonstrating the design of the MEMS gravimeter. The central proof mass is suspended from three flexures: an anti-spring pair at the bottom and a curved cantilever at the top. The anti-spring pair constrain the motion of the proof mass along the red axis. The frequency is lowered by this constraint until the cantilever pushes the motion off-axis, stabilising the MEMS device at a lower frequency.

- Figure 2: The Experimental Set-up. A schematic of the MEMS device and the shadow sensor. Both sit on an aluminium plate and are encased in a copper thermal shield. Both the MEMS device and the shield are thermally controlled. At the top left is a photograph and scanning electron microscope (SEM) image of the MEMS device. At the bottom left is a photograph of the MEMS device mounted on the optical shadow sensor with glue holding the heater and thermometer in place.

- Figure 3; The Earth Tides. The measurements of the Earth tides obtained from the MEMS device. The data has been averaged with a time constant of 240 minutes. The red line is a theoretical plot calculated with TSOFT, including an ocean loading correction. The blue line is the experimental data. The two series have a correlation coefficient of 0.86 .

- Figure 4: Drift Characteristics. 4a is a full noise time series of the tide measurement. 4b is the Allan Deviation of the series in $4 \mathbf{a}$, $4 \mathbf{c}$ is a full noise time series of the tide measurement with the tide signal removed via a regression against the theoretical data from TSOFT. 4d is the Allan Deviation of the series in 4c, 4e is a time series of the tide measurement with the tides removed and the linear drift corrected, $\mathbf{4 f}$ is the corresponding Allan deviation plot. $4 \mathbf{g}$ is the same data as $4 \mathbf{e}$ but with a 4 hour filter added. $4 \mathbf{h}$ is the Allan deviation plot of 
this filtered data.

\section{Extended data figure legends}

- Figure 5: Spring Resonant Frequency Behaviour with Tilt The resonant frequency decreases as the MEMS device gets closer to vertical due to the geometrical anti-spring effect. At $88^{\circ}$ and and $92^{\circ}$ there are minima in the plot. At this point the frequency is constant with tilt and the system displays a Hooke's law behaviour. The resonant frequency of a symmetric anti-spring would reach an instability here. This figure also demonstrates that whilst the instrument is operated at $90^{\circ}$ the resonant frequency is $2.3 \mathrm{~Hz}$, it can be lowered to $1.8-1.9 \mathrm{~Hz}$ by tilting to operate to one of the minima.

- Figure 6: The Earth Tides with Different Filtering. Figure6a presents measurements of the Earth tides obtained from the MEMS device. This is the raw data output. Figure $6 \mathrm{~b}$ presents the same data but with a 10 minute filtering time. The red lines are theoretical plots calculated by TSOFT. The blue lines are the experimental data.

- Figure 7: Quality Factor Frequency Dependence. We observe a trend of decreasing quality factor with decreasing frequency of our device. At low frequencies the internal friction of the material becomes the dominant loss mechanism. This trend has been discussed by Chin et al. 21 .

- Figure 8: Geometrical Anti-Spring Design. Figures 8a and 8b demonstrate the Hooke's-law behaviour of a straight and curved cantilever respectively. Figures $8 \mathrm{c}$ and $8 \mathrm{~d}$ demonstrate the unstable anti-spring characteristics of a 2 and 4 flexure MEMS device respectively. Figure 8e demonstrates behaviour of a 3 flexure MEMS device (see figure 1). 
Whilst a 2 or 4 flexure system reaches an instability with increasing load, a 3 flexure system regains a Hooke's law behaviour. The 3-flexure system behaves as such because it is pushed off its constrained axis by the asymmetry of the design. All of these plots were produced using Ansys finite element analysis software.

- Figure 9: Polynomial Drift. This plot demonstrates the drift in the data shortly after the vacuum pump has been turned on. A polynomial component to the drift is clearly visible. Once the vacuum system has settled, however, the drift becomes linear as demonstrated in figure $4 \mathrm{~b}$ at a level of $150 \mu \mathrm{Gal} /$ day.

- Figure 10: MEMS Device RMS Acceleration Sensitivity. Figure 10a demonstrates the RMS acceleration sensitivity in $\mu \mathrm{Gal}$, and figure $10 \mathrm{~b}$ in $\mu \mathrm{Gal}-\mathrm{dB}$. The tide signal can be observed in both plots at $10^{-5} \mathrm{~Hz}$; the peak at $2 \times 10^{-3} \mathrm{~Hz}$ is the artefact of the temperature servo discussed earlier; the microseismic peak can be observed $0.1 \mathrm{~Hz}$ and $0.2 \mathrm{~Hz}$; and the 2.3 Hz resonant frequency can be observed to the right of the plot. The plot is a composite of two data series because the temporal resolution required to record the higher frequency data would not be possible to maintain at lower frequencies.

- Figure 11: Power Spectral Density Comparison. The red series - plotted using the data from $4 \mathrm{~g}$ - is our MEMS device, demonstrating its sensitivity in the tidal frequency range. The filtering time means that the sensitivity rolls off above $10^{-4} \mathrm{~Hz}$. The black series is the Scintrex CG5, the blue series is the Micro-g Lacoste gPhone-054, the green series is the SGC026 superconducting gravimeter. The data from these three series are taken from a figure by Riccardi et. al. ${ }^{30}$ (C)Bureau International des Poids et Mesures. Reproduced by permission of IOP Publishing. All rights reserved.). The magenta series is the microseismometer by Pike et. al. (private communication by permission of the author, to be published in the 47th 
Lunar and Planetary Science Conference).

- Figure 12: Tilt Susceptibility Tests. Figure 12a demonstrates the variation in output of the MEMS device with the x-axis tilt of the sensor plotted on a secondary axis. Figure 12b shows the same for the y-axis. There is a $\mathrm{x}$-axis (in-plane MEMS tilt) a tilt sensitivity in this axis of $21.2 \mu \mathrm{Gal} /$ arc second, but in the $\mathrm{x}$-axis (out of plane MEMS tilt) the tilt sensitivity of only $0.6 \mu \mathrm{Gal} /$ arc second.

- Figure 13: Long Term Reproducibility Tests. Figures 13a and 13b are two data sets separated by approximately 4 months, with no filtering employed. During this period the vacuum chamber was evacuated and vented several times, despite this the calibration factor of the device has not changed by more than $5 \%$. 


\section{${ }_{313}$ Methods}

\section{MEMS device fabrication}

The MEMS device was fabricated from a single chip of $200 \mu \mathrm{m}$ thick silicon. The reverse side of the wafer was first coated with $2.5 \mu \mathrm{m}$ of PECVD $\mathrm{SiO}_{2}$. A $100 \mathrm{~nm}$ coating of chromium was next deposited on the top surface of the silicon using a thermal evaporator.

The MEMS device pattern was created in a layer of positive photoresist using a g-line photolithography process. The mask was a 'halo' design ${ }^{31}$ i.e. instead of etching away all of the unwanted areas of silicon, trenches were used in an outline of the structure, to keep a constant etch rate and profile over all etched areas. The halo was $10 \mu \mathrm{m}$ wide. The photoresist pattern was then used as a mask to wet etch the chrome using a nitric acid chrome etchant for $100 \mathrm{~s}$, thus etching the MEMS device proof mass pattern into the chrome. The resist was then removed ultrasonically with acetone and isopropanol, leaving the chrome etch mask in place. A $7 \mu$ m layer of $A Z^{\mathrm{R}}$-4562 photoresist was then spun onto the back of the sample and used later in making the sample free standing.

The sample was fixed to a carrier wafer (chrome side up) using a thin, spun-on layer of Crystalbond $^{\circledR} 509$ in solution with acetone. To ensure a good thermal contact the sample was weighted and left on the hotplate at $88^{\circ} \mathrm{C}$ (just above the melting point of Crystalbond ${ }^{\circledR}$ ) for 5 minutes. The sample was next placed in an Oxford Instruments PlasmaPro 100 Estrelas Deep Silicon Etch System, and Bosch ${ }^{\mathrm{TM}}$ etched $^{32}$ for 80 minutes using an $\mathrm{SF}_{6}, \mathrm{C}_{4} \mathrm{~F}_{8}$ process optimised for highly anisotropic trenches. This etch was the same depth as the silicon and stopped when it reached the $\mathrm{SiO}_{2}$ back layer. The PlasmaPro 100 Estrelas Deep Silicon Etch System allows control of the gas flow enabling processes to be tuned with negative and positive defined etch profiles. Our spring profiles are vertical to within $0.5^{\circ}$. 
To remove the sample from the carrier wafer it was heated to $88^{\circ} \mathrm{C}$ for 5 minutes, and then pushed laterally off the - now fluid - Crystalbond ${ }^{\circledR}$. The $\mathrm{SiO}_{2}$ and $A Z^{\mathrm{R}}$-4562 layers enabled this to be done without damaging the MEMS device structure. The sample was then turned upside down and placed (not affixed) to a blank piece of silicon. The residual Crystalbond ${ }^{\circledR}$ and photoresist were removed from the bottom of the sample using an $\mathrm{O}_{2}$ plasma ash. The sample was exposed to a $\mathrm{CF}_{4} / \mathrm{O}_{2}$ etchant plasma until all of the $\mathrm{SiO}_{2}$ was removed, making the sample free standing.

\section{Geometrical Anti-Spring Design}

Our MEMS device is comprised of a proof mass, suspended from three curved cantilevers/flexures.

To better understand the physical characteristics of this system we first discuss these flexures individually. Consider a cantilever, clamped at one end, and free to move at the other. A proof mass mounted on the moving end will oscillate with a frequency that depends on the geometry of the cantilever, and the Young's modulus of the material from which it is made. The proof mass will oscillate along an arc, defined by the length of the flexure. The system will behave as a Hooke's law spring, with a linear relationship between force and displacement. This behaviour can be observed in figure 8a. A curved single cantilever also behaves in the same manner, as seen in figure $8 \mathrm{~b}$.

To create an anti-spring, one can take two such curved cantilevers and attach them at a central pivot point. A proof mass mounted at this point will no longer be able to trace out an arc as it oscillates. Instead - because of the symmetrical forces applied by the two identical cantilevers - its motion will be constrained along a vertical axis (as presented in figure 1). It is this constraint that forces the spring constant to change as the displacement increases. Instead of observing a linear relationship between force and displacement, a non-linear behaviour is found. This behaviour can be observed in figure $8 \mathrm{c}$. This now means that the spring gets softer with increasing displacement. 
A four flexure anti-spring system is a simple extension of a two-flexure system. Here, a second pair of cantilevers are placed below the first pair, this allows a non-point source proof mass to be suspended. The behaviour of the spring is still non-linear, and is displayed in figure $8 \mathrm{~d}$. The behaviour is identical to that of a two flexure system, except the system can support twice the mass.

Both the two and four anti-spring systems can be used to create oscillators that have low resonant frequencies. When the limits of $k / m$ are pushed to create the lowest resonant frequency possible, however, these systems become unstable. They become unstable because the motion is so well constrained along its vertical axis, the spring gets softer and softer until it can no longer support the weight of the proof mass. This behaviour can be observed in figures $8 \mathrm{c}$ and $8 \mathrm{~d}$; as the force increases, the displacement increases rapidly. A stable resonant frequency is imperative for a useful relative gravimeter, therefore this instability would create problems if used for the design of a MEMS gravimeter. It would require the use of a closed-loop feedback system.

Our MEMS device utilises a novel three-flexure anti-spring system, with one flexure of the upper pair of cantilevers removed (see figure 1). In the first instance, the device behaves as a four-flexure anti-spring: it gets rapidly softer as the displacement of the proof mass increases. The anti-spring behaviour is maintained while the proof mass moves along its vertically constrained axis. The asymmetry of the system, however, means that the device does not stay constrained along the anti-spring constraining axis. The single upper flexure ultimately tilts the proof mass marginally away from the constraining axis. As the motion is pulled from this axis, the anti-spring trend is halted and the device regains a Hooke's law behaviour, where $d F / d z=$ constant. This behaviour can be observed in figure $8 \mathrm{e}$ where the gradient of force vs displacement reaches a minimum at $z=0.6$. This means that the device assumes a constant spring constant at the maximum stiffness value that we have demonstrated to be stable over many months (as demonstrated by figure 13 . 


\section{Optical shadow sensor}

The proof mass motion is measured using an optical shadow sensor $\underline{14}$. Using a fused silica ' $\mathrm{C}$ 'shaped support structure, a red LED (powered at $0.3 \mathrm{~mW}$ ) was shone onto a split photodiode, with the MEMS device proof mass mounted in between. The change in intensity incident on the photodiode resulting from the motion of the proof mass shadow was then used as a measure of the motion. The split photodiode was made from two $5 \mathrm{~mm}$ by $10 \mathrm{~mm}$ planar silicon photodiodes, and wired to give a differential output. A split photodiode was used so that at the nominal position of the proof mass the output signal was zero. This allowed maximal amplification without saturation of the measurement instrumentation. The LED signal was modulated (at a frequency of $107 \mathrm{~Hz}$ with a 50:50 duty cycle) to reduce the $1 /$ f noise in the output signal. The modulation was carried out by turning the LED on and off with an HP 33120A square wave signal generator. A precision current stabalising resistor (displayed in figure 2 maintained the LED drive current, this was heat sunk to the fused silica 'C'-shaped structure. The current output from the photodiode was first converted into a voltage using an $S R S S R 570$ current-to-voltage converter, band-passed between $3 \mathrm{~Hz}$ to $100 \mathrm{~Hz}$, and amplified by a factor of $10^{6} \mathrm{~V} / \mathrm{A}$. This amplified signal was then de-modulated via an analogue lock-in amplifier (Femto $L I A-M V$-200) referenced from the signal generator. The lock-in amplified the signal with a gain of 10 and undertook readings with a time constant of 3 s. This analogue signal was passed through an $S R S S R 560$ low pass filter $0.03 \mathrm{~Hz}$ to remove aliasing and filter seismic noise, before being digitised via a 16 bit, $12 \mathrm{~dB} /$ octave, analogue-todigital converter (National Instruments $M$ Series 6229) and recorded by a computer with a $24 \mathrm{~s}$ time constant. Analogue signals were used to reduce digitisation noise that would have occurred if a digital signal had been amplified by this magnitude.

The shadow sensor has a readout noise floor of $\leq 10 \mu \mathrm{Gal}$ at the sampling frequency of $0.03 \mathrm{~Hz}$, 
and a dynamic range of $\sim 50 \mu \mathrm{m}$. A large dynamic range is required because of the large initial displacement $(0.8 \mathrm{~mm})$ of the proof mass when it is tilted to its vertical operating orientation, thus making initial alignment of the MEMS device difficult. Although the maximum peak-to-peak displacement of the proof mass caused by the tides is only $16 \mathrm{~nm}$, the proof mass also oscillates at its resonant frequency by up to $100 \mathrm{~nm}$ due to seismic ground motion. A high dynamic range is also useful to measure this signal, which is ultimately removed from the data by averaging with a $0.03 \mathrm{~Hz}$ filter in the readout electronics.

\section{Temperature control}

The control loops used to maintain the temperature of the system were proportional integral derivative (PID) control mechanisms, written in Labview. Temperatures were monitored using a four-terminal measurement of small platinum resistors, via two Keithley 2000 digital mulitmeters. A four-terminal measurement eradicates contact resistance by driving the thermometer with a current and measuring the voltage across it. This removes the temperature sensitivity of external wires. Low temperature coefficient Manganin ${ }^{\circledR}$ wires were used for these connections to minimise parasitic thermal conduction. One platinum resistor was placed on the outer frame of the MEMS device and three were placed equidistantly around the copper shield. Wire wound resistors were used as the heating mechanism to feedback into the system; again, one of these was placed on the MEMS device frame and three around the shield. The output signal to the heaters was sent via a National Instruments (USB 6211) card, and the heaters were powered with non-inverting amplifiers with a capability to power up to $100 \mathrm{~mA}$. All circuitry and instrumentation used to amplify and measure the output signal, and to measure and control the system temperature, were selected for their high thermal stability. This entire configuration was constructed in a vacuum chamber with a pressure of $\leq 10^{-5}$ mTorr. 
429

Although PID control was implemented for the MEMS device and the shield, there were other components with variations that could not be actively controlled. Namely the room temperature that coupled into the data via a temperature sensitive lock-in amplifier, and intensity variations of the LED that were monitored using a monitor photodiode. There was also an offset, and a linear drift of under $150 \mu \mathrm{Gal}$ per day once the system had been left evacuated for over a week. This drift term is due to stress in the silicon flexures. Like all mechanical systems, application of stress leads to anelasticity which causes creep and drift over long timescales. Our device also shows polynomial drift which decays away approximately one week after evacuating the apparatus. The polynomial drift is likely due to adsorbed water on the surface layer of silicon, and could be mitigated against by baking out the system before evacuation. Figure 9 demonstrates this initial polynomial drift. The data were therefore regressed against the temperature measurements listed above, the drift offset and the intensity. This regression - carried out in Matlab with the mregg tool - identified correlations between the output data and these parameters, and removed any resulting correlated trends from the final data. Floor tilt and power variation of the LED were also monitored, but neither had any discernable effect on the signal and were therefore not regressed.

The correlation coefficient, $R$, between the averaged theoretical and experimental tide data was calculated using Matlab's 'corrcoeff' function. An $R$ value of 0.86 was produced, for the plot presented in figure 3. To check the level of significance of our experimental data we compared it to the correlation of the noise alone. We created 10,000 random permutations of our data set and calculated the correlation coefficient for each with respect to the theoretical data. This set of $R$ values were plotted as a histogram. This histogram had a distribution with a mean value of zero and a standard deviation of 0.008 . The $\mathrm{R}$ value from the un-randomised data is $114 \sigma$ from this 
distribution suggesting the correlation is real to an extremely high degree of confidence.

Figure 10 is a plot of the RMS acceleration sensitivity of the device over its full spectral range. The tide signal can be observed at $1 \times 10^{-5} \mathrm{~Hz}$. The peak at $10^{-3} \mathrm{~Hz}$ is an artefact of the temperature servo. Between $0.1 \mathrm{~Hz}$ and $0.2 \mathrm{~Hz}$ the micro-seismic peak can be recognised, its presence indicates that the device is also a sensitive seismometer. Past observations - made from Scotland in February to March 2000 - of the microseismic peak ${ }^{28}$ confirm the validity of our observation. At $2.3 \mathrm{~Hz}$ the primary resonant mode of the MEMS device generates a large peak due to excitation from seismic noise. This plot was used to calculate the sensitivity of the MEMS device. To find a sensitivity in $\mu \mathrm{Gal} / \sqrt{H z}$, it is just necessary to read off the acceleration sensitivity at the point where the data crosses $1 \mathrm{~Hz}$ on the horizontal axis. We believe that the value of $40 \mu \mathrm{Gal} / \sqrt{\mathrm{Hz}}$ is an overestimate of the true sensitivity of the device because at $1 \mathrm{~Hz}$ the influence of both the primary resonance of the device and the micro-seismic peak are significant.

\section{Tilt variation}

Although tilt did not have an effect on the tide measurement, we are interested to know at what point tilt would become an issue. Figure 12 presents two plots of an experiment used to asses the effect of tilt on our device. Inside the vacuum tank, the MEMS device was mounted vertically and aligned with the tilt sensor. The y-axis of the tilt sensor was aligned with the plane of the MEMS device, with the $\mathrm{x}$-axis perpendicular to this (see Fig. 11). Figure 12a demonstrates the induced tilts of the tank in both $\mathrm{x}$ and $\mathrm{y}$ axes in arc seconds. Figure $12 \mathrm{~b}$ demonstrates the corresponding change in the output of of the device in $\mu \mathrm{Gal}$. There is a strong correlation between the $\mathrm{y}$ axis variation and the voltage output, giving a tilt sensitivity in this axis of $21.2 \mu \mathrm{Gal} /$ arcsec. There is less sensitivity to the $\mathrm{x}$-axis tilt with a tilt sensitivity of only $0.6 \mu \mathrm{Gal} /$ arcsec.

The $\mathrm{x}$-axis tilt sensitivity is low because in the vertical configuration, the spring resumes a 
Hooke's law response as observed in Fig. 5, for which the x-axis tilt variation is plotted against the resonant frequency (the acceleration sensitivity of the device is proportional to the square of the resonant frequency). Ultimately the spring could be tuned to operate with even less variation with tilt in this axis if it were positioned to operate at one of its minima. Alternatively the flexures could be made marginally thicker to shift the minimum in resonant frequency to $90^{\circ}$, this was not carried out because the device did not show sufficient tilt sensitivity to cause concern. The y-axis variation is larger because the device has a mode of oscillation in which the proof mass tilts and pivots about the upper cantilever flexure.

When vertical, the device would need to be levelled with an accuracy limited by the y-axis sensitivity (i.e. less than 2 arc seconds to maintain the current sensitivity) to make repeatable measurements in different locations. This accuracy of levelling is achievable with a simple surveyors bubble level.

\section{Temporal Reproducibility Tests}

Figure 13 demonstrates two short data sets separated by nearly four months. These were used as a test of the temporal stability of the device. To convert the raw voltage output of the device into a unit of acceleration, a calibration factor was required. By comparing the experimental (blue) data in Fig. $13 \mathrm{a}$ with that in $13 \mathrm{~b}$ we were able to test whether the calibration factor had drifted over time. The same calibration factor has been used to make both of these plots. By averaging the data and changing the calibration factor of Fig. 13b, it was found that a change in the calibration factor of $5 \%$ made the fit to the tide theory (red) data noticeably worse. Changes smaller than this were not possible to resolve. We therefore believe that if the calibration factor has changed, it has done so by no more than 5\%. During this time period, the vacuum tank was vented and evacuated several times, the MEMS was moved around each time. This is an important feature of 
a device that could eventually be used in the field.

\section{Applications}

MEMS gravimeters have significant industrial applications. Given their small size and low cost, they could be used for down bore-hole exploration in the oil and gas industry ${ }^{33}$ and utilised to monitor well drainage. Such devices could also be utilised for environmental monitoring, where networks of sensor arrays could monitor sub surface water levels ${ }^{34}$, or to determine the location of historic landfill sites. The security industry is an area for which low cost/small form factor gravimeters would also be a transformative technology to detect subterranean tunnels ${ }^{35,36}$, or imaging of cargo containers where high spatial resolution via numerous sensors is an advantage ${ }^{37}$. MEMS gravimeters could also be used in civil engineering. At present in many of the UK victorian cities the placement of utilities is only accurate on maps to within 15 metres of land marks such as trees, fences or buildings. There have been trials of the Scintrex CG5 and MEMS based arrays would offer an exciting opportunity. Gravimetry is already used in volcanology and can be used to help predict eruptions. Networks of small, low-cost gravimeter arrays could revolutionize the way volcano gravimetry is carried out ${ }^{22,25,24}$.

A field prototype is currently being developed at Glasgow that will be the size of a tennis ball and require a power supply of under $1 \mathrm{~W}$. A powerless getter pump will be used to maintain vacuum, both the thermal control and the optical readout will be on-chip; tilt levelling will be included, and all of the read-out and control software will be run on a micro-controller. 


\section{Additional references from methods section}

[31] Pike, W. Analysis of sidewall quality in through-wafer deep reactive-ion etching. Microelectronic Eng. 73-74, 340-345 (2004).

[32] Laermer, F. \& Schilp, A. Method of anisotropic etching of silicon. US patent number: $5,501,893$ (1996).

[33] Rim, H. \& Li, Y. Advantages of borehole vector gravity in density imaging. Geophysics. 80, G1-G13 (2015).

[34] Bauer-gottwein, P., Christiansen, L \& Rosbjerg, D. Informing hydrological models with ground-based time-lapse relative gravimetry : potential and limitations. GRACE, Remote Sensing and Ground-based Methods in Multi-Scale Hydrology (Proceedings of Symposium JH01)., 187-194 (2011).

[35] Romaides, A. J. et al. A comparison of gravimetric techniques for measuring subsurface void signals. J.Phys. D. Appl. Phys. 34, 433-443 (2001).

[36] Butler, D. K. Microgravimetric and gravity gradient techniques for detection of subsurface cavities. Geophysics 49, 1084-1096 (1984).

[37] Kirkendall, B., Li, Y. \& Oldenburg, D. Imaging cargo containers using gravity gradiometry. IEEE Transactions on Geoscience and Remote Sensing 45, 1786-1797 (2007).

[38] Panisova, J. \& Pasteka, R. The use of microgravity technique in archaeology: A case study from the St. Nicolas Church in Pukanec, Slovakia. Contributions to Geophysics and Geodesy 39, 237-254 (2009). 


\section{${ }_{537}$ Acknowledgements}

${ }_{538}$ The work was funded by the Royal Society Paul Instrument Fund and STFC grant number 539 ST/M000427/1. The authors would like to thank Dr Matthew Pitkin for advice on complet540 ing significance tests on the data, Dr William Cunningham for advice on finite element modelling, 541 Mr Michael Perreur-Lloyd and Mr Russell Jones for their help in rendering 3D images of the ap${ }_{542}$ paratus, and the staff and other users of the James Watt Nanofabrication Centre for help and support in undertaking the MEMS fabrication. 


\section{Author contributions}

- R.P.M. led the methodology of the etch process for the MEMS gravimeter and worked with G.D.H. on the development of the MEMS gravimeter. With G.D.H., he enhanced the longterm, low-noise stability of the entire system, taking the tide data and performing the computational analysis. He led writing the manuscript.

- A.S. led the methodology of the MEMS mask fabrication. With R.P.M he took the tide measurements in early 2015 and performed computational analysis of the MEMS gravimeter.

- D.J.P. supervised the design of the MEMS device fabrication process and with G.D.H. came up with the concept for a MEMS gravity sensor.

- J.H. developed the methodology of utilising geometric anti-springs for the MEMS gravimeter system and provided critical review/commentary on the manuscript.

- S.R. was responsible for the resources which were necessary to complete the project and provided critical review/commentary on the manuscript.

- G.D.H. had the initial concept of a MEMS gravimeter together with D.J.P. He had oversight of the design, fabrication and testing of the gravimeter, via the supervision of R.P.M and A.S. Together with R.P.M., he characterised and enhanced the low noise performance, resulting in the measurement of the tides. He was responsible for funding acquisition of the work. 


\section{${ }_{561}$ Author information}

- The research data relevant to this letter are stored on the University of Glasgow's Enlighten Repository, DOI: http://dx.doi.org/10.5525/gla.researchdata.213

- Reprints and permissions information is available at www.nature.com/reprints

- The authors have no competing financial interests.

- Correspondence and requests for material should be addressed to giles.hammond@glasgow.gla.ac.uk or r.middlemiss.1@research.gla.ac.uk. 
${ }_{568}$ Extended data 


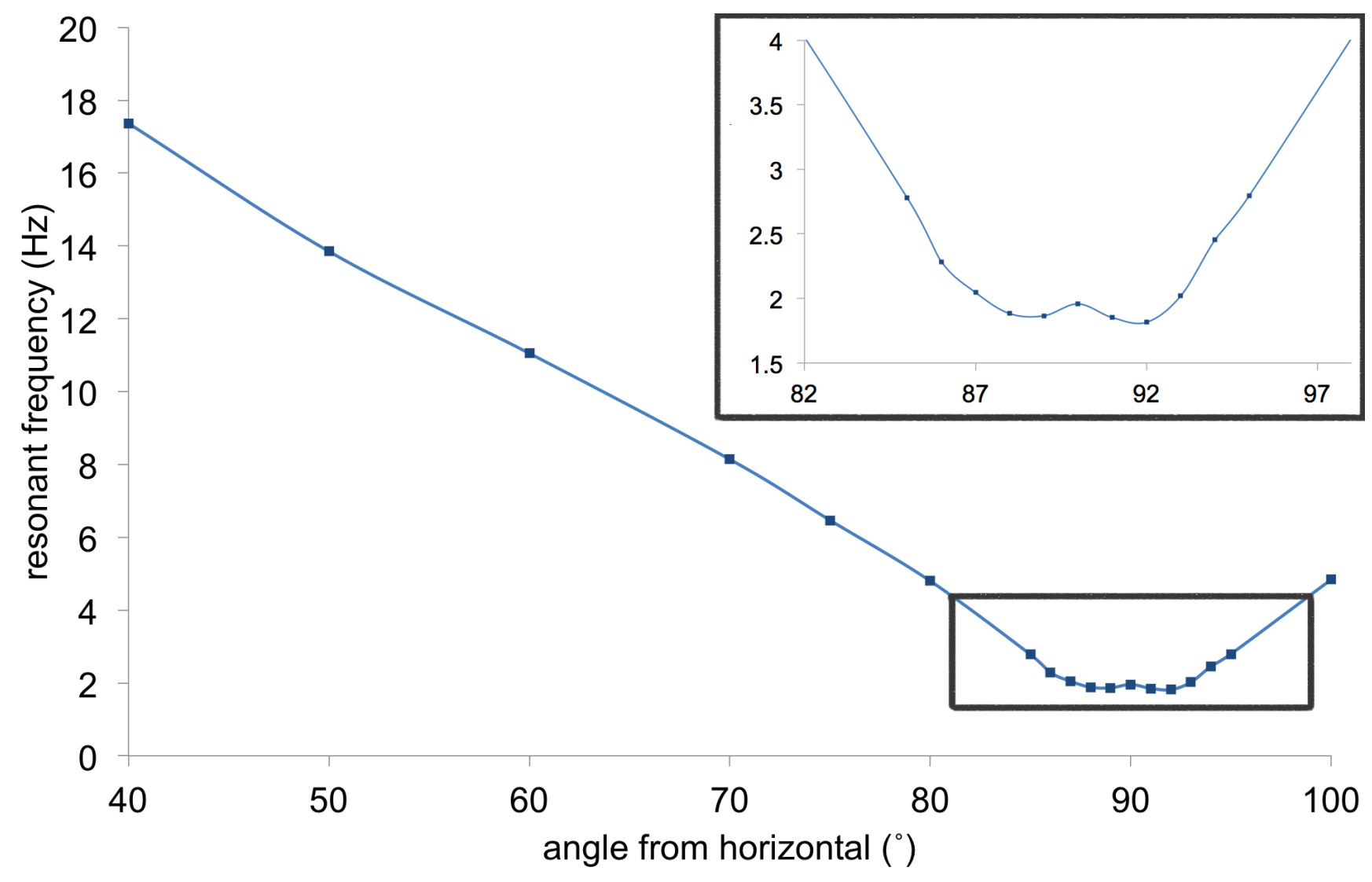

Figure 5 


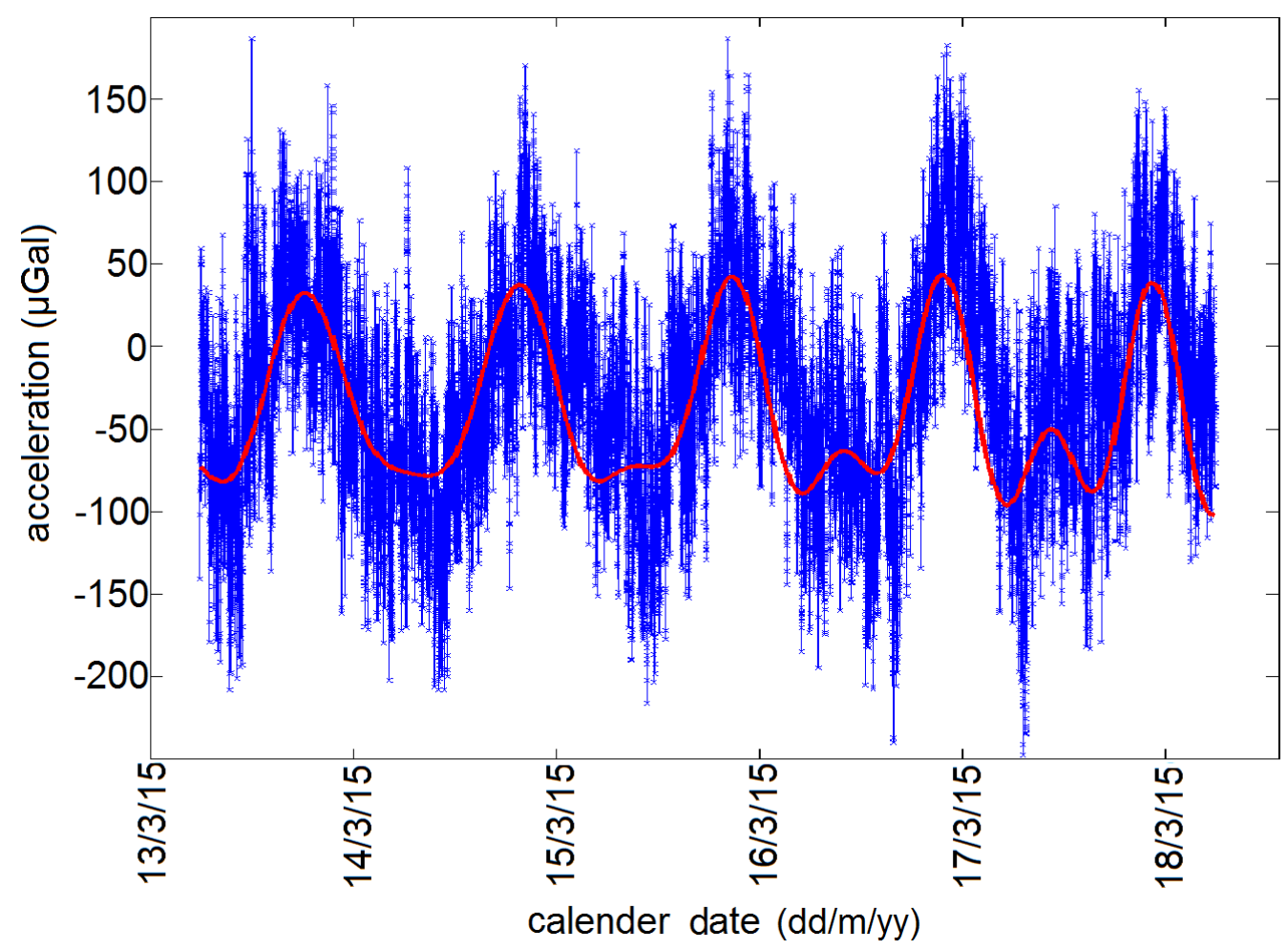

(a)

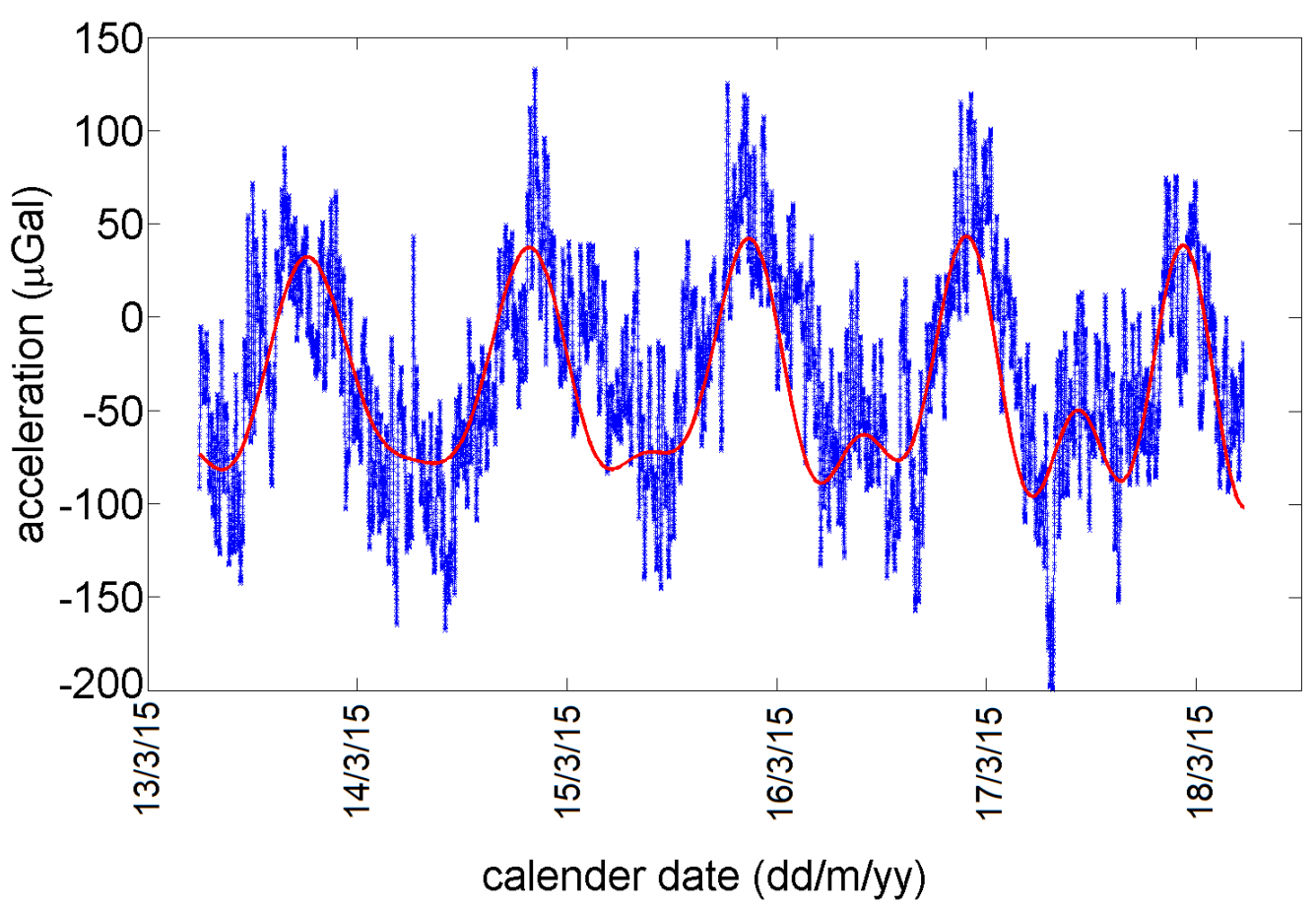

(b)

Figure 6 


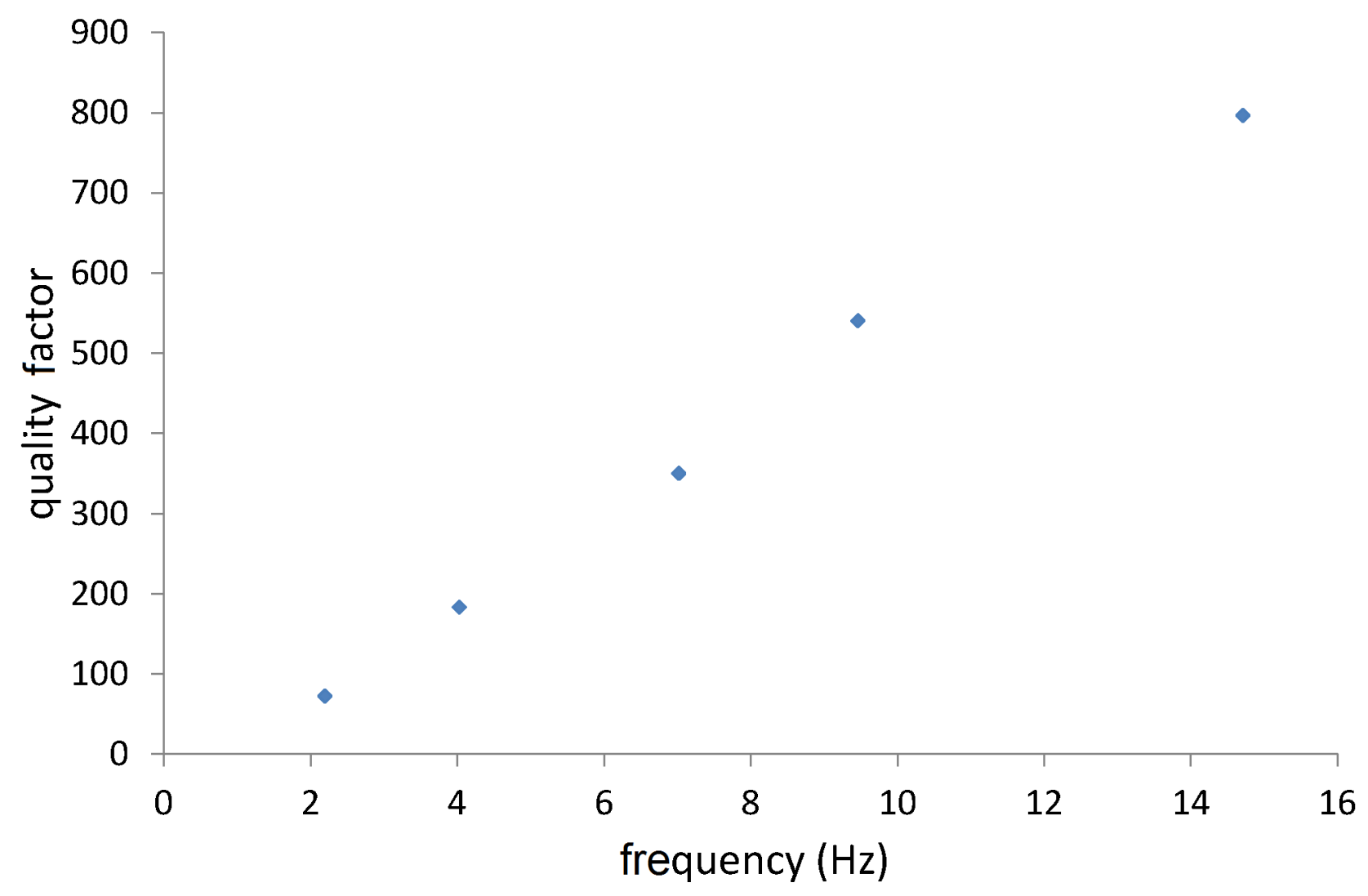

Figure 7 

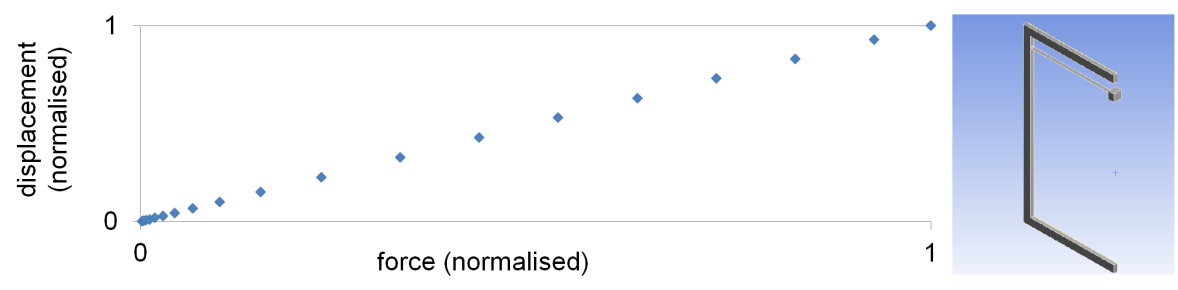

(a)
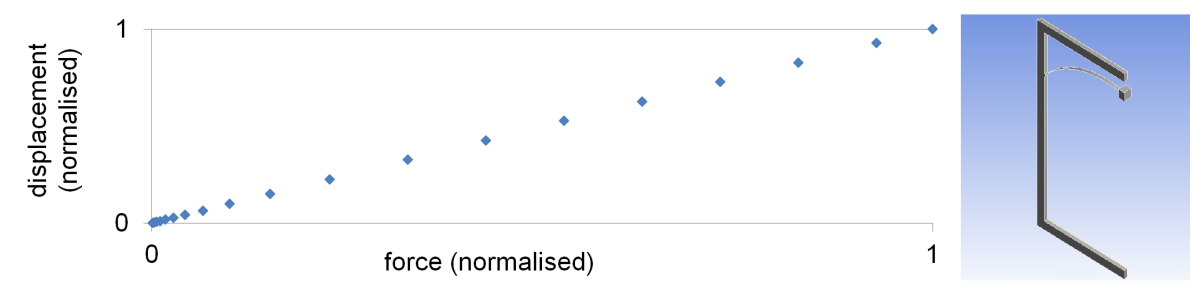

(b)

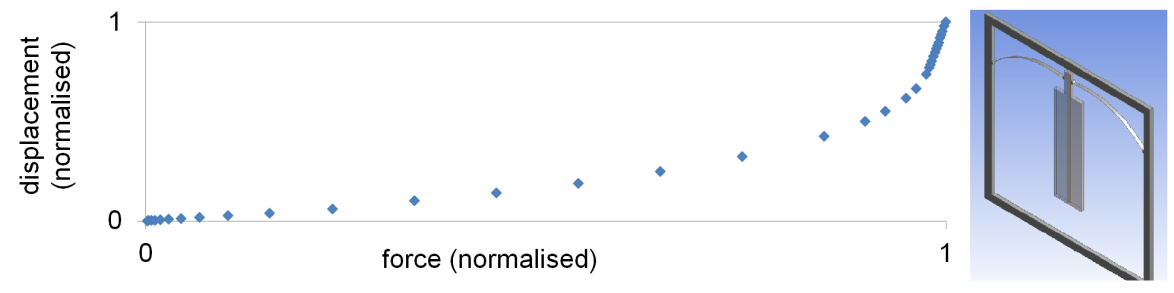

(c)

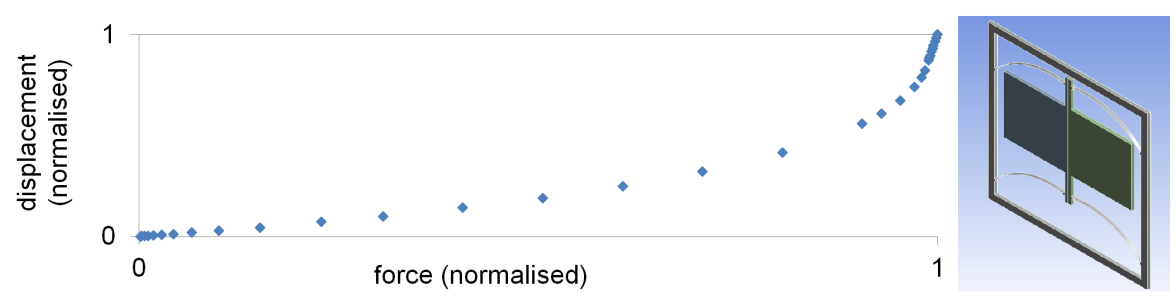

(d)

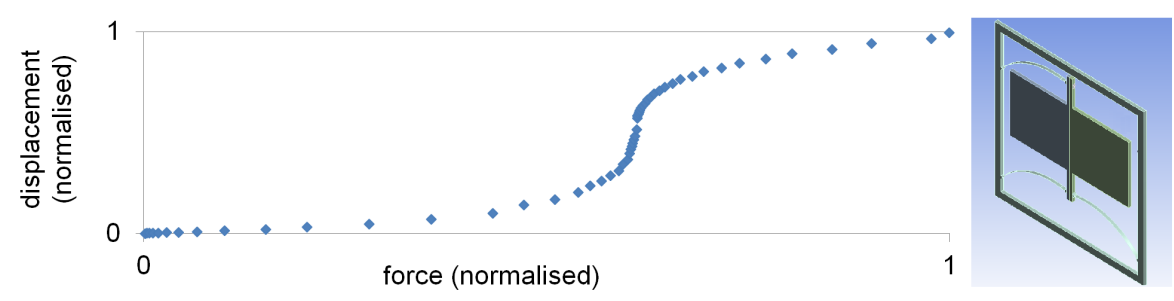

(e)

Figure 8 


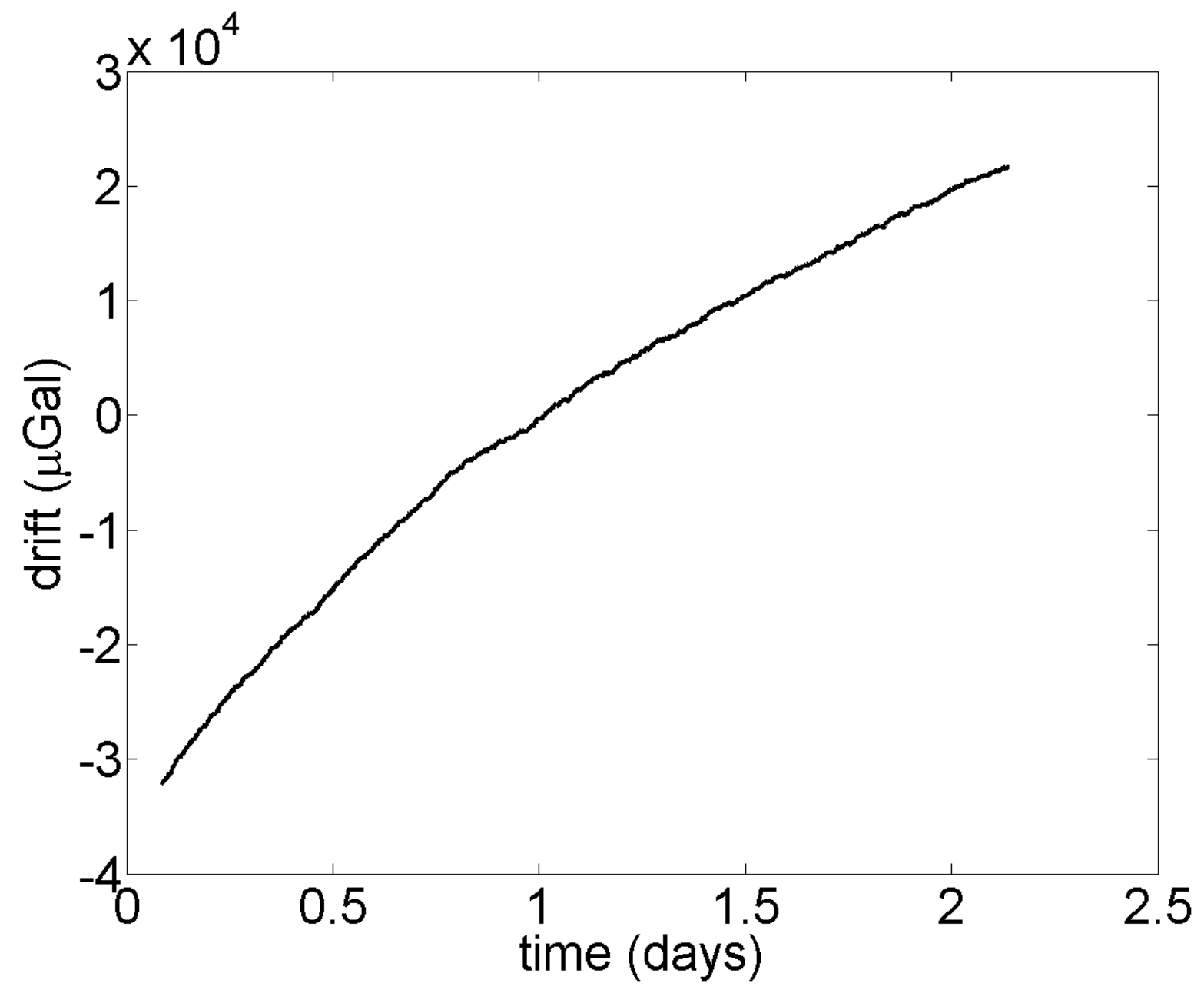

Figure 9 


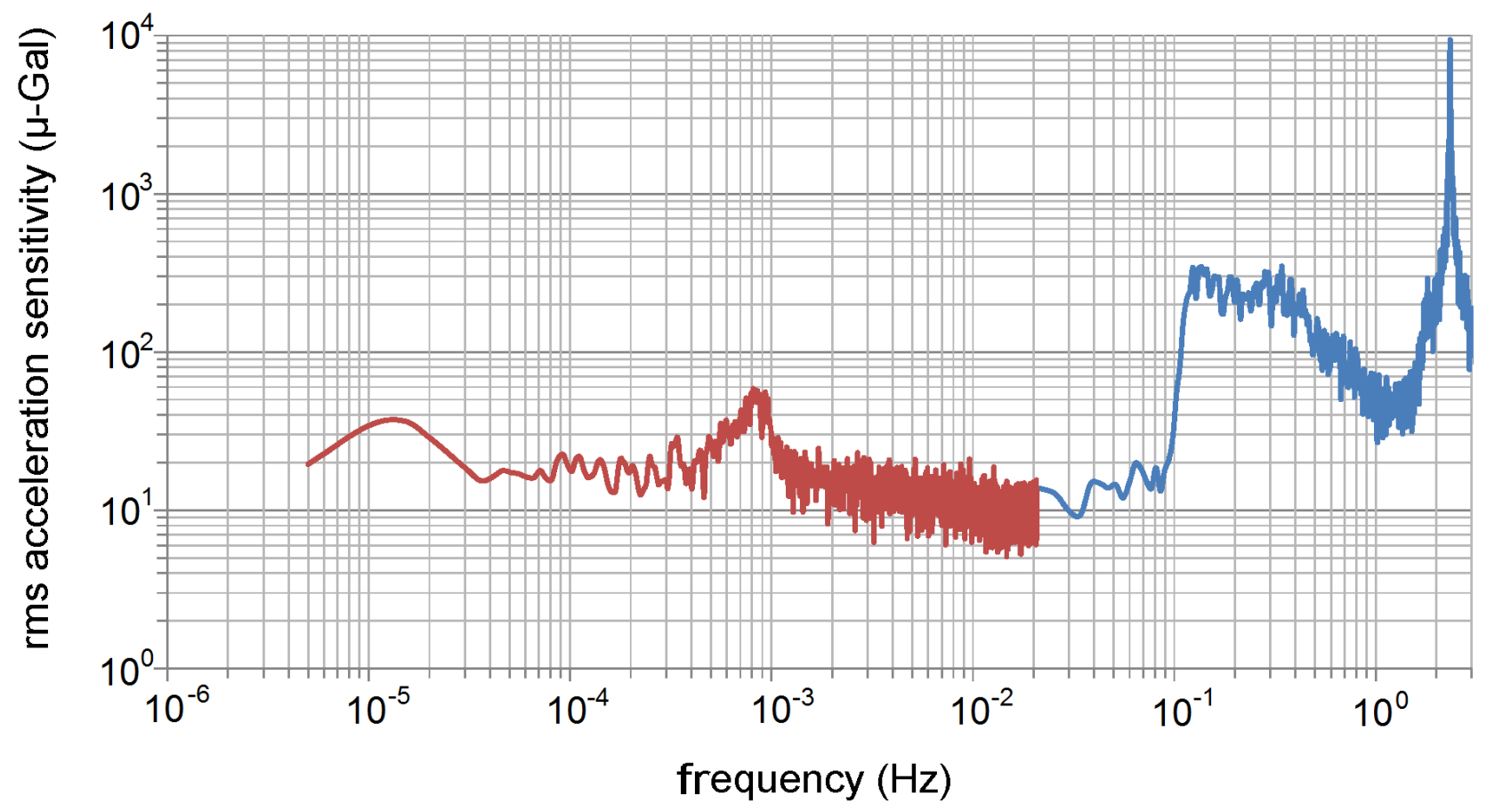

(a)

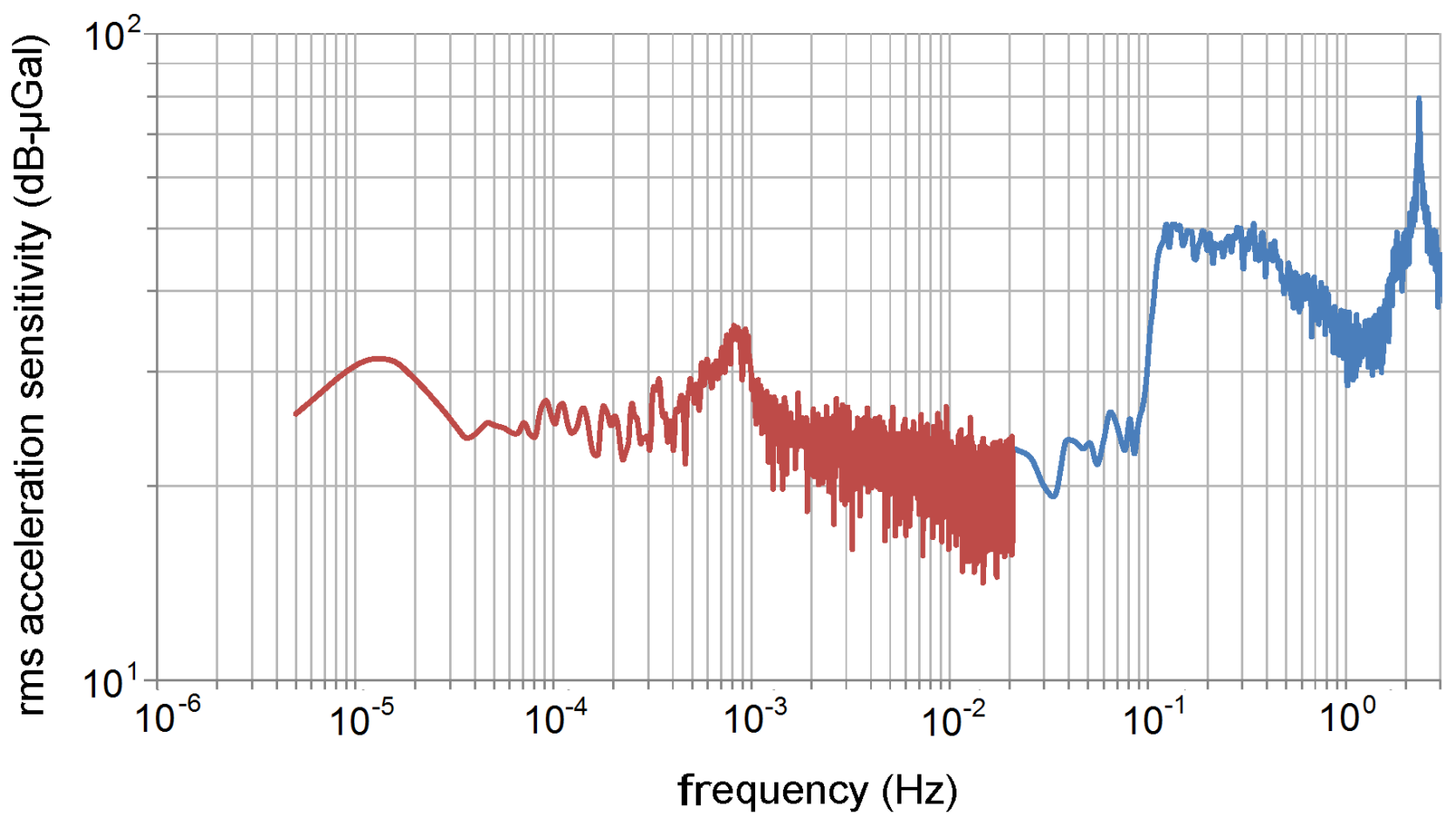

(b)

Figure 10 


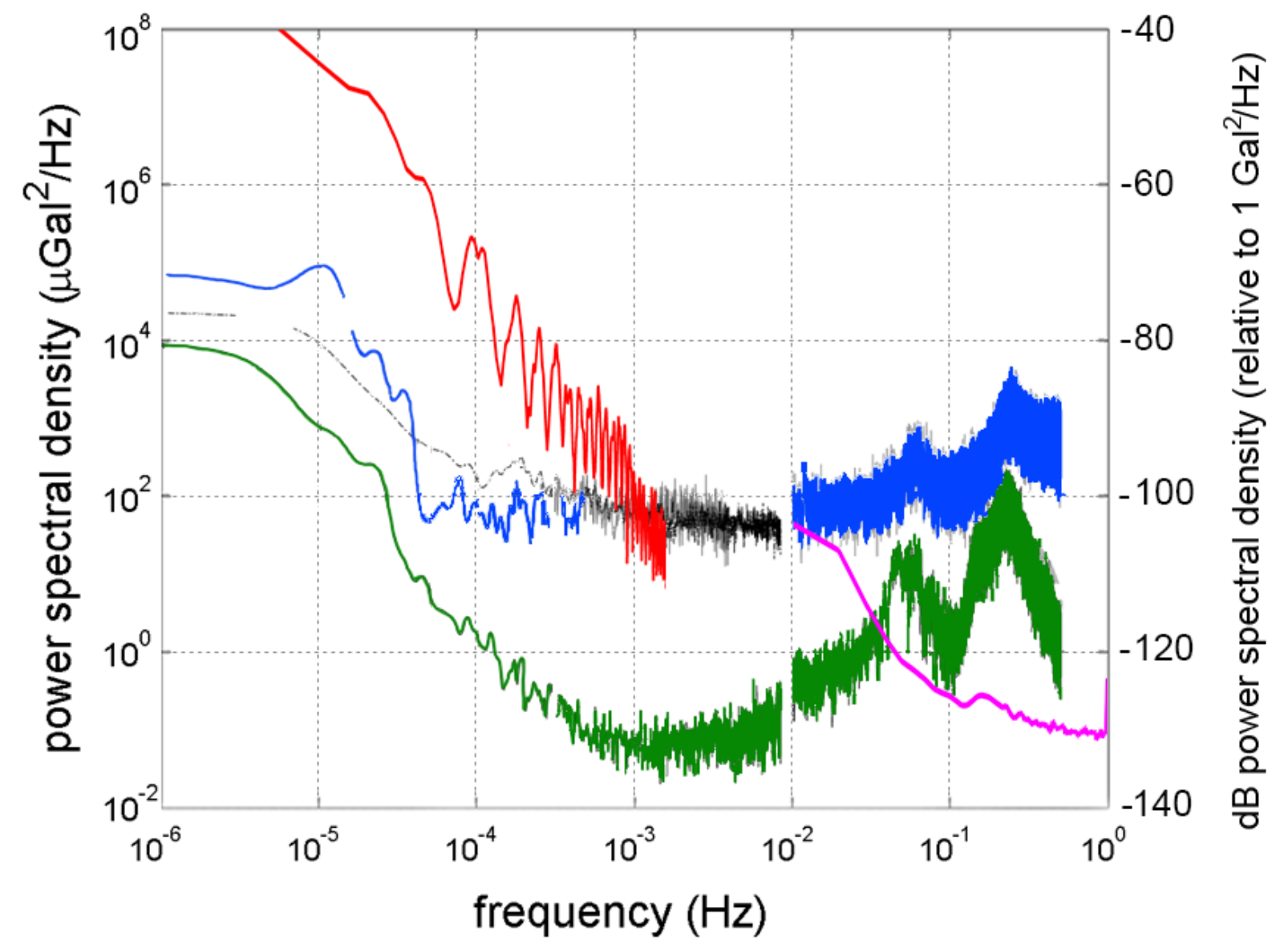

Figure 11 


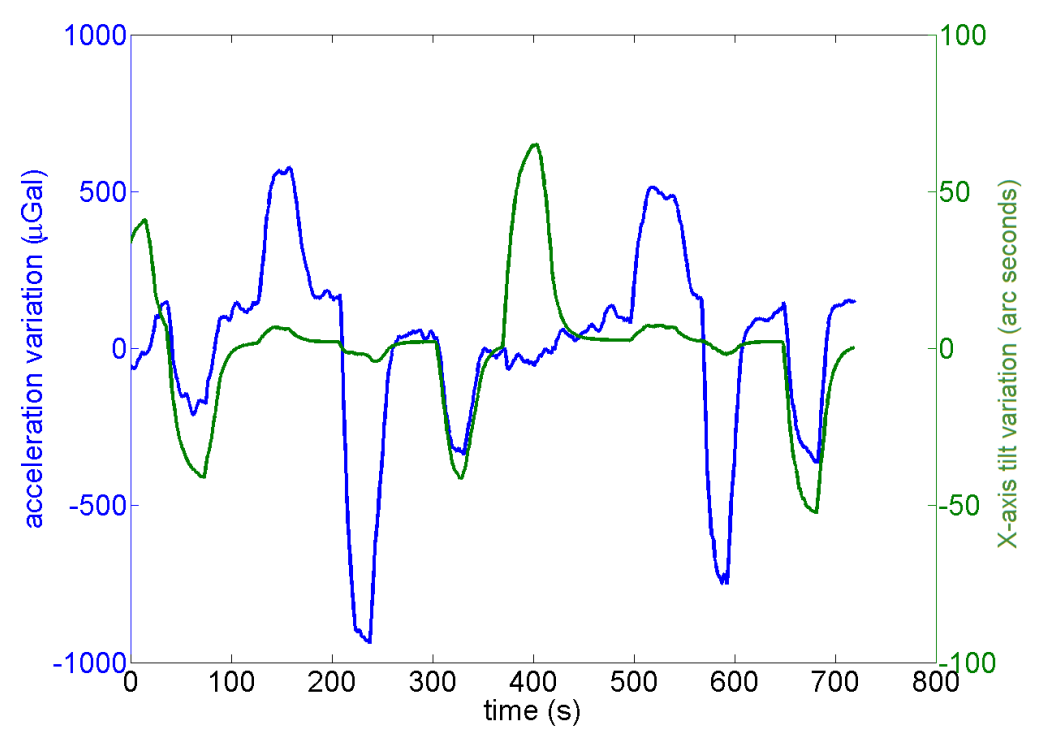

(a)

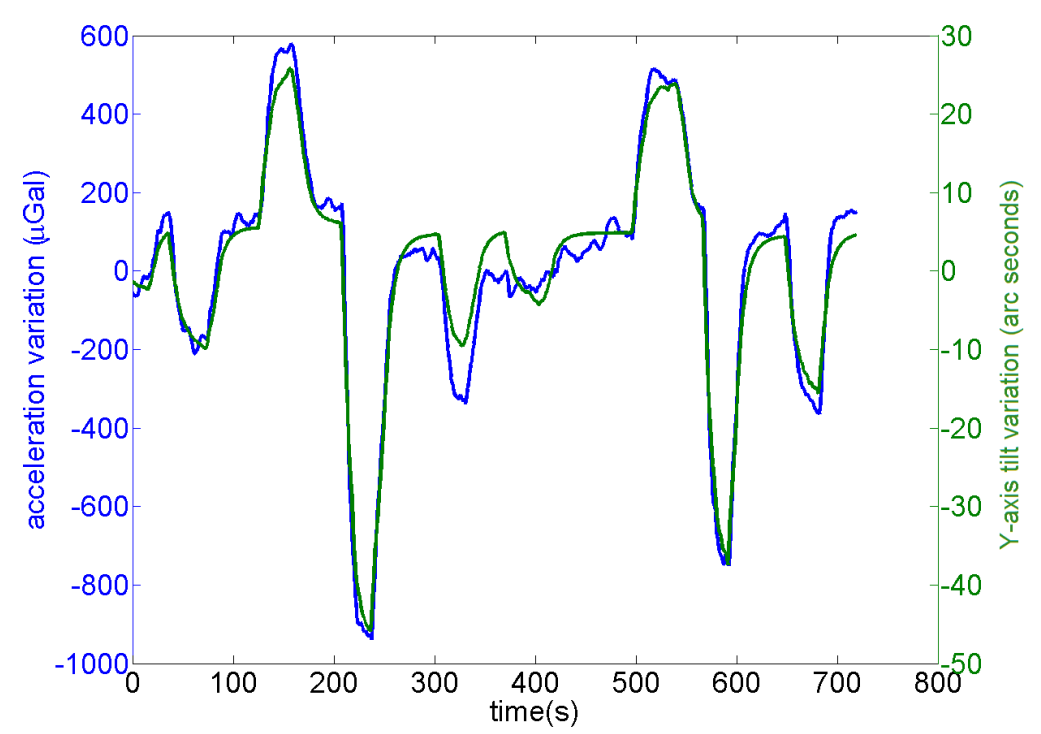

(b)

Figure 12 


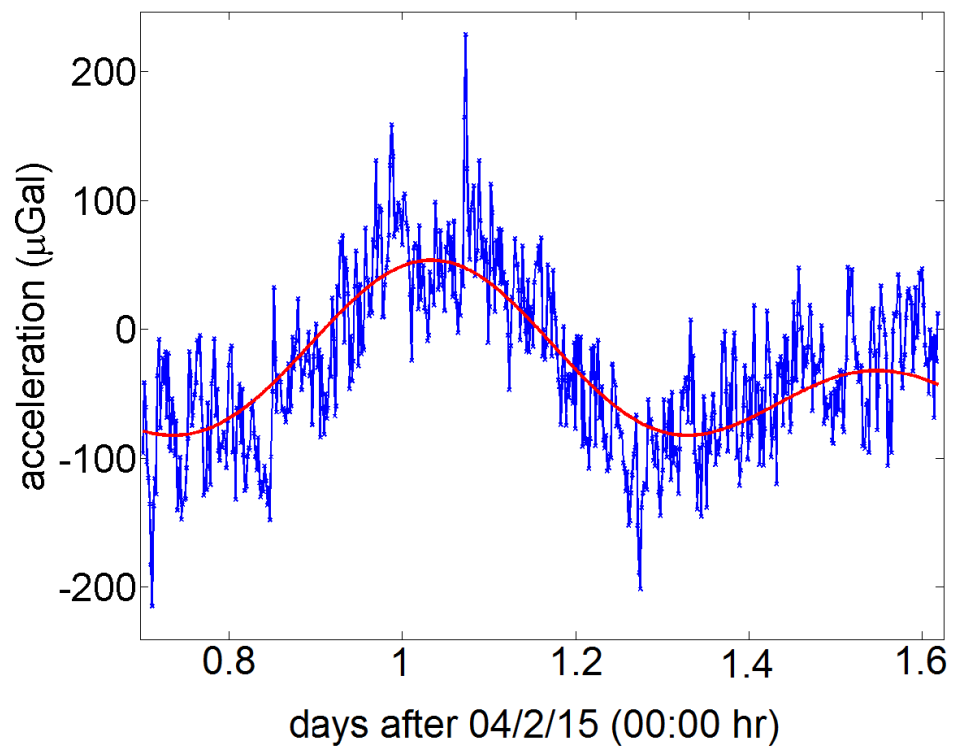

(a)

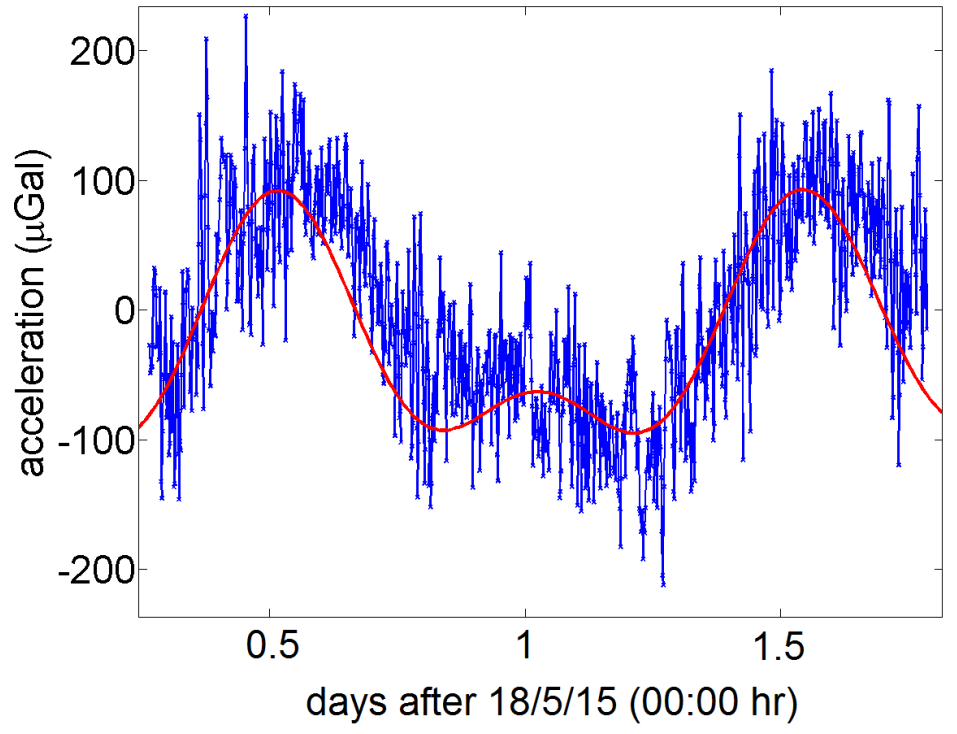

(b)

Figure 13 


\begin{tabular}{|c|c|c|c|c|c|}
\hline Device & Technology & $\begin{array}{c}\text { Sensitivity } \\
\text { at } 1 \mathrm{~Hz}\end{array}$ & $\begin{array}{c}\text { Stability in } \mu \mathrm{Hz} \\
\text { Regime }\end{array}$ & $\begin{array}{c}\text { Resonant } \\
\text { Fre- } \\
\text { quency }\end{array}$ & Use \\
\hline \hline Scintrex CG5 & $\begin{array}{c}\text { Fused } \\
\text { Quartz }\end{array}$ & $2 \mu \mathrm{Gal}$ & $0.5 \mathrm{mGal} /$ day & $3 \mathrm{~Hz}$ & Gravimetry \\
\hline Krishnamoorthy $\left.^{8}\right]$ & MEMS & $17 \mu \mathrm{Gal}$ & N/A & $36 \mathrm{~Hz}$ & Seismology \\
\hline Quietseis $^{9}$ & MEMS & $15 \mu \mathrm{Gal}$ & N/A & $800 \mathrm{~Hz}$ & Seismology \\
\hline Pike1 $^{10}$ & MEMS & $2 \mu \mathrm{Gal}$ & N/A & $11 \mathrm{~Hz}$ & Seismology \\
\hline Glasgow MEMS & MEMS & $40 \mu \mathrm{Gal}$ & $0.14 \mathrm{mGal} /$ day & $2.31 \mathrm{~Hz}$ & Gravimetry \\
\hline
\end{tabular}

Table 1: Comparison of Acceleration Sensors 